\title{
Dectin-2-induced CCL2 production in tissue-resident macrophages ignites cardiac arteritis
}

\author{
Chie Miyabe, ${ }^{1}$ Yoshishige Miyabe, ${ }^{1}$ Laura Bricio-Moreno,, Jeffrey Lian,, ${ }^{1}$ od A. Rahimi, ${ }^{1}$ Noriko N. Miura, ${ }^{2}$ Naohito Ohno, ${ }^{2}$ \\ Yoichiro Iwakura, ${ }^{3}$ Tamihiro Kawakami, ${ }^{4}$ and Andrew D. Luster ${ }^{1}$ \\ 'Center for Immunology and Inflammatory Diseases, Division of Rheumatology, Allergy and Immunology, Massachusetts Ceneral Hospital, Harvard Medical School, Boston, Massachusetts, USA. \\ ${ }^{2}$ Tokyo University of Pharmacy and Life Sciences, Tokyo, Japan. ${ }^{3}$ Center for Animal Disease Models, Research Institute for Biomedical Sciences, Tokyo University of Science, Chiba, Japan. \\ ${ }^{4}$ Division of Dermatology, Tohoku Medical and Pharmaceutical University, Sendai, Japan.
}

\begin{abstract}
Environmental triggers, including those from pathogens, are thought to play an important role in triggering autoimmune diseases, such as vasculitis, in genetically susceptible individuals. The mechanism by which activation of the innate immune system contributes to vessel-specific autoimmunity in vasculitis is not known. Systemic administration of Candida albicans water-soluble extract (CAWS) induces vasculitis in the aortic root and coronary arteries of mice that mimics human Kawasaki disease. We found that Dectin-2 signaling in macrophages resident in the aortic root of the heart induced early CCL2 production and the initial recruitment of CCR2 ${ }^{+}$inflammatory monocytes (iMos) into the aortic root and coronary arteries. iMos differentiated into monocyte-derived dendritic cells (Mo-DCs) in the vessel wall and were induced to release IL-1 $\beta$ in a Dectin-2/Syk/NLRP3 inflammasome-dependent pathway. IL-1 $\beta$ then activated cardiac endothelial cells to express CXCL1 and CCL2 and adhesion molecules that induced neutrophil and further iMo recruitment and accumulation in the aortic root and coronary arteries. Our findings demonstrate that Dectin-2-mediated induction of CCL2 production by macrophages resident in the aortic root and coronary arteries initiates vascular inflammation in a model of Kawasaki disease, suggesting an important role for the innate immune system in initiating vasculitis.
\end{abstract}

\section{Introduction}

Kawasaki disease (KD), first described as an acute, febrile, mucocutaneous lymph node syndrome in 1967 (1), is the most common systemic vasculitis of children and infants. $\mathrm{KD}$ is recognized as the leading cause of acquired heart disease among children in developed countries (2). KD primarily involves the coronary arteries, but vasculitis can occur at various sites throughout the body (3). Coronary artery abnormalities, including dilatation and aneurysms, are the most serious complications that develop in approximately $25 \%$ of untreated cases, which can lead to myocardial ischemia, infarction, and death (4). High-dose intravenous immunoglobulin (IVIG) therapy reduces the risk of coronary artery lesions (5); however, approximately $10 \%-15 \%$ of the KD patients do not respond to IVIG, and appropriate treatment for these cases is not established (6). Therefore, understanding the pathogenesis of $\mathrm{KD}$ is critical for the rational design of improved therapies for preventing the cardiac complications of $\mathrm{KD}$.

Although the etiology of $\mathrm{KD}$ is not known, an infectious agent has been suspected due to the reported seasonal peak during winter and spring seasons (7). Moreover, a number of studies have linked KD with exposure to freshly cleaned carpets, habitation near a body of water, and humidifier use. Various bacterial and viral agents have been implicated as potential causes of $\mathrm{KD}$, but

Conflict of interest: The authors have declared that no conflict of interest exists. Copyright: @ 2019, American Society for Clinical Investigation.

Submitted: July 25, 2018; Accepted: May 29, 2019; Published: July 29, 2019.

Reference information: J Clin Invest. 2019;129(9):3610-3624.

https://doi.org/10.1172/JCI123778. no proof has emerged to incriminate a single pathogen (8). In addition, there is also growing evidence supporting genetic contributions to the susceptibility to KD (9). These findings suggest that infectious triggers in genetically susceptible individuals likely play an important role in the development of KD vasculitis.

Because of inherent difficulties in studying human patients with vasculitis, animal models have served as important tools for improving our understanding of the basic mechanisms underlying the pathogenesis of vasculitis (10). Over the last few decades, several murine models of KD have been developed that share pathological features with the human disease, including the Candida albicans water-soluble extract-induced (CAWS-induced) vasculitis model $(11,12)$, the Lactobacillus casei cell wall extract-induced (LCWEinduced) vasculitis model (13), and the NOD1 ligand-induced vasculitis model (14). Although animal models are not identical to human disease and it is not established that Candida causes KD, previous studies have suggested that exposure to wind-borne Candida species might be one trigger for $\mathrm{KD}$ (15-17). In addition, it has been reported that anti-Candida cell wall $\beta$-glucan serum antibody titers are higher in KD patients compared with normal controls (18). These reports suggest potential physiological relevance for the CAWS-induced vasculitis model. Interestingly, all of these models have in common strong innate immune system activation by pathogen-associated molecular patterns (PAMPs). Pattern recognition receptors (PRRs), including TLRs, retinoic acid-inducible gene Ilike receptors (RLRs), and C-type lectin receptors (CLRs), recognize PAMPs and initiate the innate immune response (19).

Here we explored the mechanism by which the initiation of an innate immune response leads to vasculitis using the CAWS- 
induced murine model of vasculitis. CAWS consists of Candida albican's cell wall, including $\alpha$-mannan and $\beta$-glucan (20). Both CLRs and TLRs play a role in Candida recognition. Among the CLRs, Dectin-1 recognizes $\beta$-glucans, whereas Dectin- 2 recognizes $\alpha$-mannans in fungal cell walls $(21,22)$. Both Dectin- 1 and Dectin-2 are glycosylated type II transmembrane proteins that are mainly expressed on myeloid cells, and induce cytokines and reactive oxygen species to protect hosts from fungal infection through activation of a spleen tyrosine kinase (Syk)/CARD9/NF- $\kappa$ B pathway in DCs and macrophages (23). Whereas Dectin-1 has a tyrosine-based activation motif-like (ITAM-like) motif (hemITAM) in its intracellular region, Dectin-2 associates with an ITAM-containing $\mathrm{Fc}$ receptor $\gamma(\mathrm{FcR} \gamma)$ signaling chain. Among the TLRs, TLR2 recognizes phospholipomannan, while TLR4 recognizes O-linked mannosyl residues of the Candida cell wall, which are involved in the induction of host immune response to Candida (24).

Here we describe what we believe is a novel mechanism whereby the innate immune response ignites autoimmune vessel-specific inflammation. We show that CAWS antigen is preferentially deposited in the adventitia of the aortic root where it is recognized by Dectin-2 expressed on resident macrophages in the adventitia. Dectin-2-activated resident macrophages produce CCL2, which induces the recruitment of inflammatory monocytes (iMos) into the aortic root, the initial step required for the induction of aortic root and coronary artery vasculitis resembling KD. iMos differentiate into monocyte-derived DCs (Mo-DCs) and produce IL-1 $\beta$ via a Dectin-2/NF- $\kappa$ B/NAPL3 inflammasome pathway, resulting in chemokine production in the inflamed vessel amplifying neutrophil and iMo recruitment.

\section{Results}

CAWS induces inflammatory cell recruitment into the aortic root area. CAWS induces marked inflammatory cell infiltration into the aortic root area (Figure 1A). To clarify the temporal and spacial distribution of inflammatory cell infiltration into the heart during the development of CAWS-induced arteritis, we analyzed the number of inflammatory cells in coronal sections of the entire heart before and on days 1, 7, and 28 after beginning CAWS injection on day 0 by IHC using Ly6G/Ly6C to stain neutrophils and iMos and F4/80 to stain resident macrophages (Figure 1, B and C). The vast majority of Ly6G/Ly6 $\mathrm{C}^{+}$and $\mathrm{F} 4 / 80^{+}$cells accumulated in the aortic root area during the development of vasculitis, peaking on day 28 , with a small number of Ly6G/Ly6 $\mathrm{C}^{+}$cells transiently observed in the myocardium on day 1 (Supplemental Figure 1). These results suggest that CAWS injection induces inflammatory cell recruitment mainly into the aortic root area, as has been previously reported $(11,12,25)$. These results indicate that, although we have used the entire heart for subsequent FACS analysis, it is presumed that the majority of inflammatory cells detected by flow cytometry were from the aortic root area of the heart.

To define the initiating events promoting coronary arteritis, we performed a detailed kinetic analysis of myeloid cell subsets recruited into the heart after 5 daily i.p. injections of CAWS into C57BL/6 WT mice. Whole-heart cell suspensions were obtained after enzyme digestion and analyzed by flow cytometry on days $0,1,2,4,7$, and 28 after the first dose of CAWS. After gating on live $\mathrm{CD}_{4} 5^{+}$cells and excluding cells expressing CD90.2, CD19, and NK1.1 to remove T cells, B cells, and NK cells, a sequential gating strategy was employed based on the differential expression of CD11b, Ly6G, CD64, MerTK, Ly6C, MHCII, and CD11c to define myeloid cell subsets $(26,27)$. This allowed the identification of cardiac macrophages $\left(\mathrm{CD} 64^{+} \mathrm{MerTK}^{+}\right)$, neutrophils $\left(\mathrm{CD} 11 \mathrm{~b}^{+} \mathrm{Ly}_{6 \mathrm{G}^{+}}\right)$, DCs $\left(\mathrm{CD} 11 \mathrm{c}^{+} \mathrm{MHCII}^{+}\right)$, Ly6 $\mathrm{C}^{\text {hi }}$ monocytes (Ly6 $\left.\mathrm{C}^{\text {hi }} \mathrm{MHCII}^{-}\right)$, and Mo-DCs $\left(\mathrm{Ly}_{6} \mathrm{C}^{\mathrm{lo} /}-\mathrm{MHCII}{ }^{+}\right.$) in the heart (Figure 1D). Using this gating strategy, we found that there was a burst of Ly6 $\mathrm{C}^{\text {hi }}$ monocyte and neutrophil infiltration on day 1 (initial phase) after CAWS injection, followed by a decrease by day 2 . Thereafter, the number of Mo-DCs started to increase on day 7 (middle phase), followed by a massive influx of neutrophils on day 28 (late phase) (Figure 1E).

To determine the contribution of circulating cells to the increase in immune cells recovered from the heart following CAWS injection, a parabiosis experiment was performed. CD $45.1^{+}$ and CD $45.2^{+}$mice were cojoined for 2 weeks to allow for the establishment of a joined circulation followed by the injection of CAWS into both parabionts (Figure $1 \mathrm{~F}$ ). Mice were harvested 2 weeks after the initial CAWS injection and the ratio of $\mathrm{CD} 45^{+}$cells derived from the host and partner was approximately 60:40 in the heart, which was the same ratio as observed in blood. This demonstrates that the inflammatory cells found in the heart after CAWS injection were recruited from the circulation (Figure 1G). The chimerism of each myeloid cell type was also approximately 60:40 in the heart (Figure 1, H and I). These data show that the immune cells within the inflamed cardiac tissues were mainly recruited from the blood.

CCL 2 is induced in heart and promotes CCR $2^{+}$monocyte recruitment. Given the rapid infiltration of innate immune cells after CAWS injection, we next examined chemokine expression in the entire heart during the development of arteritis. Chemokine mRNA levels in the heart were induced differently over time following CAWS injection (Supplemental Figure 2). On day 1 after the first CAWS injection, several monocyte-attracting chemokines, including the CCR2 ligands CCL2, CCL7, and CCL12, were induced. CCR2 ligands peaked at day 1, while neutrophil-attracting chemokines, such as the CXCR2 ligands CXCL1 and CXCL2, peaked at day 28. These data suggest that during the course of CAWS-induced coronary inflammation, monocytes expressing CCR2 are recruited into the heart at early time points, while CXCR2-expressing PMNs are recruited into the heart at later time points. A detailed time course of CCL2 expression demonstrated a transient increase at day 1 after CAWS injection (Figure 2A). Remarkably, CCL2 was exclusively expressed in the heart on day 1 after CAWS injection, and was not detected in other organs at this time point, including lung, spleen, kidney, and liver (Figure 2B). Other CCR2 ligands, such as CCL7 and CCL12, were also exclusively induced in the heart 1 day following CAWS injection, while other chemokines tested were not specifically induced in the heart (Supplemental Figure 3).

To determine the role of CCR2 in monocyte trafficking from the blood into the heart, we performed adoptive transfer competitive trafficking experiments using WT and $\mathrm{Ccr} 2^{-1-}$ monocytes. WT monocytes were purified from LysM-GFP mice, in which expression of the green fluorescent protein (GFP) is driven by the lysozyme M promoter, resulting in GFP-positive neutrophils and monocytes (28). Ccr2 $2^{-/-}$monocytes were purified from CCR2-red fluorescent protein-knockin mice, which lack CCR2 in $C c r 2^{R F P / R F P}$ 
A

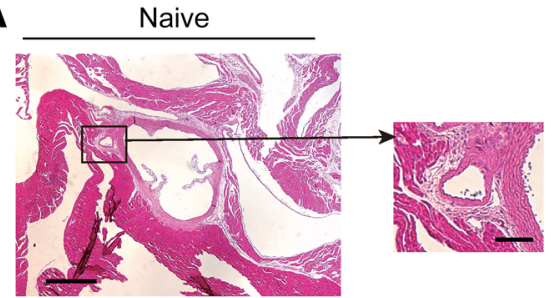

B
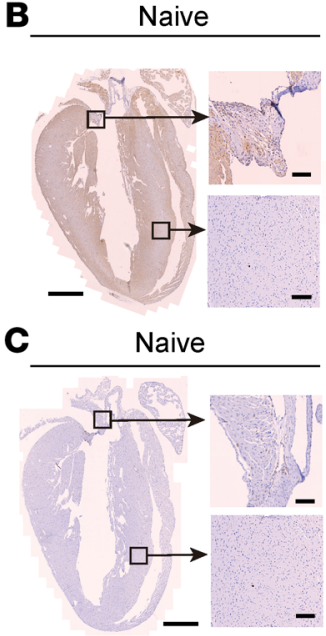

C

D Live CD45+Lin ${ }^{-} \quad$ CD11 b ${ }^{+}$Ly6G cells

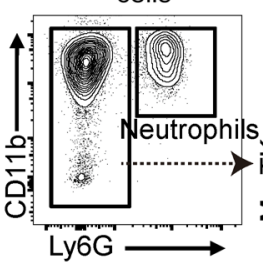

힌

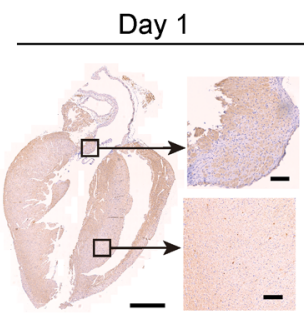

Day 1
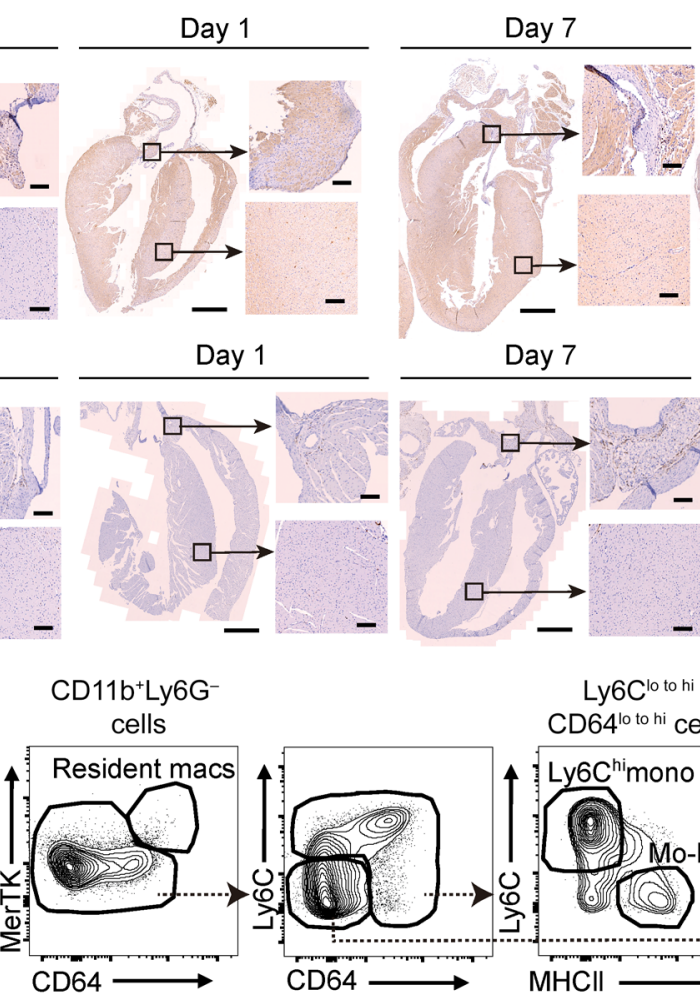

Day 7
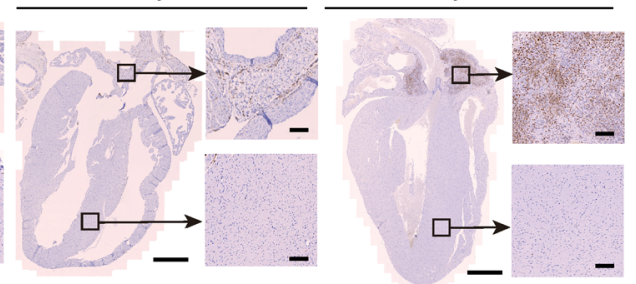

\section{E}

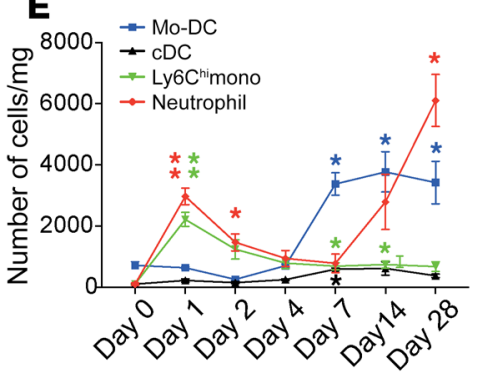

$\mathbf{F}$

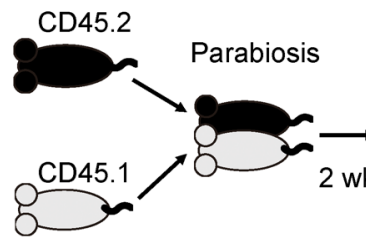

Ly6 $6 \mathrm{C}^{\text {lo to hi }}$

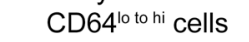

CD11b $b^{10}$ Ly6G $^{10}$ cells
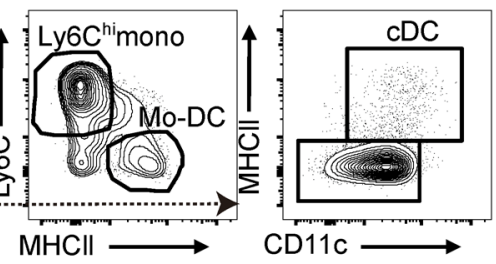

G
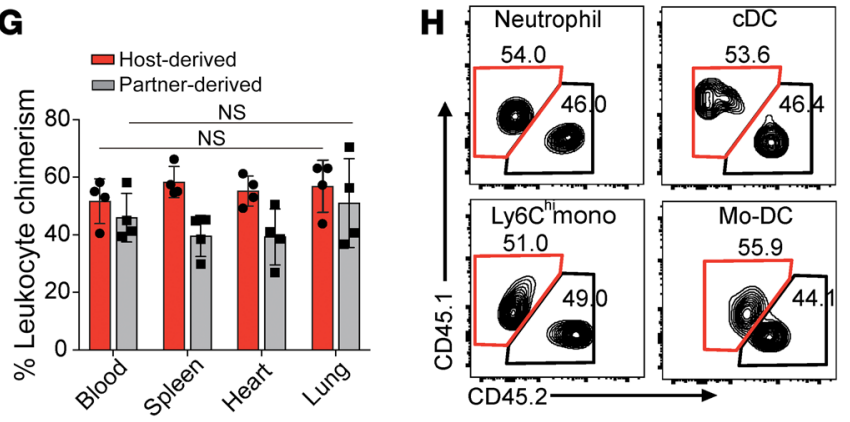

CAWS i.p. to

both mice

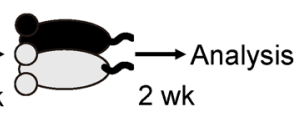

I

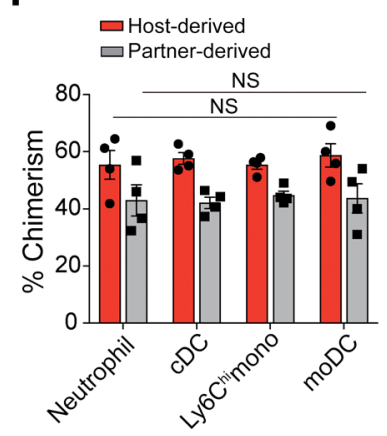

Figure 1. CAWS induces inflammatory monocyte recruitment into the heart on day 1. (A) Representative H\&E-stained horizontal section of the aortic root area from WT naive mice or on day 28 after 5 daily i.p. injections of CAWS beginning on day 0 . Low-power field shows aortic root area and high-power field shows the ostium of the coronary artery within the aortic root. Scale bars: $400 \mu \mathrm{m}$ (low-power images) and 100 $\mu \mathrm{m}$ (high-power images). (B and C) Coronal section isolated from naive or CAWS-injected WT mice (on day 1 , 7, or 28) stained with anti-Ly6C/Ly6C (B) or anti-F4/80 (C) for IHC. Cropped high-power-field images show aortic root (upper panel) or myocardium (lower panel). Scale bars: $1 \mathrm{~mm}$ (low-power images) and $100 \mu \mathrm{m}$ (high-power images). (D) Representative FACS analysis of cardiac neutrophil, macrophage, DC, and monocyte subsets recovered from CAWSinjected mice on day 1 after the first CAWS injection. One representative of 3 independent experiments. (E) Kinetics of absolute cell numbers of the indicated immune cell subset per mg of heart isolated during the course of CAWS-induced vasculitis (mean \pm SEM, $n=4-5$ mice per time point, ${ }^{*} P<0.05,{ }^{* *} P<0.01$ versus day 0 , using unpaired 2 -tailed Student's $t$ test). (F) Schematic protocol for parabiosis experiment. (C) Tissue chimerism was analyzed in parabiotic pairs by measuring the frequency of host-derived leukocytes (CD45.1+) and partner-derived leukocytes (CD45.2+) on day 14 after CAWS injection (mean $\pm \mathrm{SEM}, n=4$ mice). NS indicates statistically identical in the indicated organs among host-derived cells or partner-derived cells using ordinary 1-way ANOVA with Tukey's post hoc test. (H) Representative FACS plots of each myeloid cell subset in the parabiotic mouse hearts on day 14 after the first CAWS injection. (I) Quantitation of chimerism for the indicated myeloid cell subsets (mean $\pm \mathrm{SEM}, n=4$ mice). All values were statistically identical in indicated cell subsets among host-derived cells or partner-derived cells using ordinary 1-way ANOVA with Tukey's post hoc test. CDC, cardiac DC. 
A

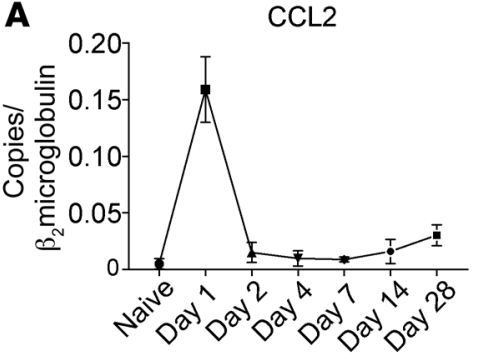

D

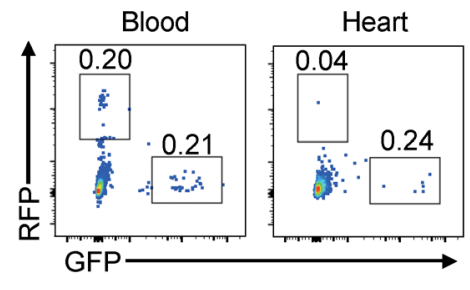

G

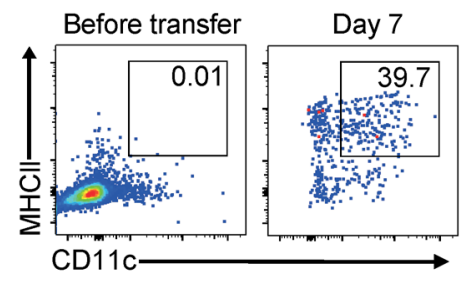

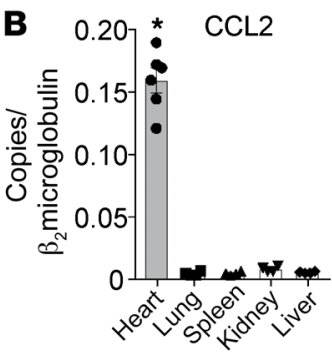

C

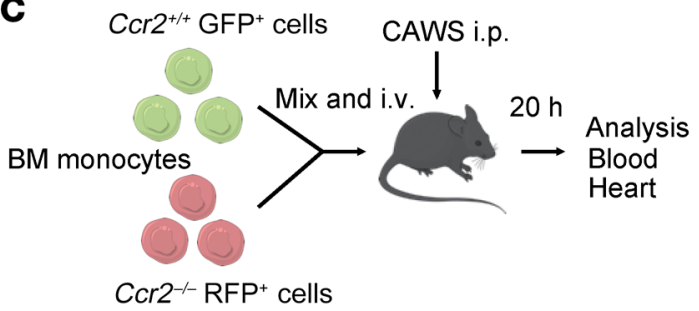

F GFP $^{+}$BM monocytes CAWS i.p. $\times 5$ times i.v. $\times 5$ times

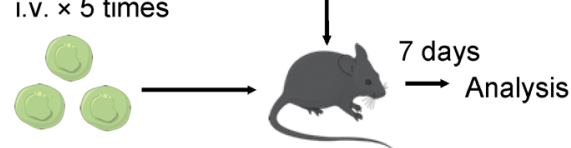

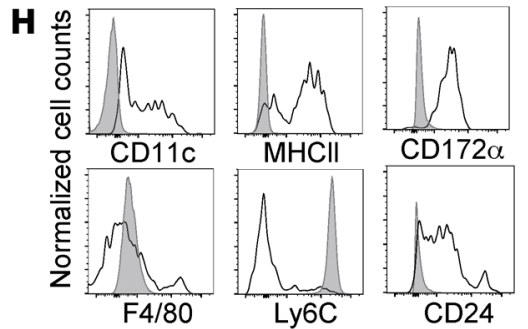
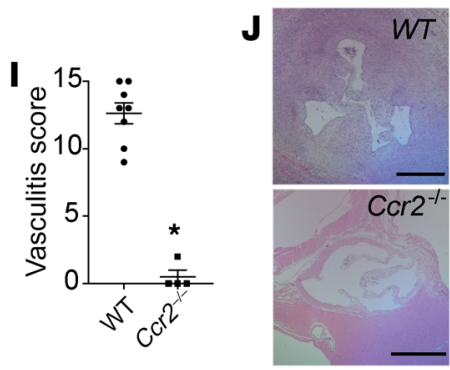

Figure 2. CCL2 is induced in the heart on day 1 and promotes CCR2+ monocyte recruitment. (A) Kinetics of CCL2 mRNA expression in the heart following CAWS injection. (B) CCL2 expression in various organs on day 1 after CAWS injection (mean \pm SEM, $n=4$ mice per group, ${ }^{*} P<0.001$ versus lung, spleen, kidney and liver). (C) Schematic of co-adoptive transfer of WT and $\mathrm{Ccr}^{-1-}$ bone marrow-derived monocytes (BMDMs) into WT mice followed by CAWS injection. Equal numbers of $\mathrm{CCr}^{+/+} \mathrm{GFP}^{+}$and $\mathrm{CCr}^{-1-} \mathrm{RFP}^{+} \mathrm{BMDMs}$ were mixed and intravenously transferred into WT recipient mice along with CAWS injection daily for 5 days. Peripheral blood and heart of recipient mice were analyzed 20 hours after cell transfer. (D) Representative FACS plots of the indicated tissues 20 hours after adoptive transfer of BMDMs and CAWS injection. $\mathrm{Ccr}^{2^{++}} \mathrm{GFP}^{+}$and $\mathrm{Ccr}^{-{ }^{--}} \mathrm{RFP}^{+}$cells in the heart tissue are shown. Numbers indicate percentages of live $\mathrm{CD} 45^{+}$cells. (E) Ratio of $C \mathrm{Cr} 2^{+/+} \mathrm{GFP}+$ cells $/ \mathrm{Ccr} 2^{-1-}$ RFP+ cells in the indicated tissues (mean $\pm \mathrm{SEM}, n=6$ mice, ${ }^{*} P<0.05$ versus blood). (F) Schematic of adoptive transfer of GFP+ BMDMs into WT mice followed by CAWS injection. (C) Representative FACS plots of the CD45+CFP+ live BMDM cell population before adoptive transfer and those recovered from the heart 7 days after CAWS injection. CAWS-injected recipient mice were adoptively transferred with GFP+ BMDMs (once daily for 5 days). (H) Representative histograms of the GFP+ live cell population before adoptive transfer (filled gray) and those recovered from heart 7 days after CAWS injection (black line). (I) Histological vasculitis scores were determined in WT and Ccr2-- mice on day 28 (mean $\pm \mathrm{SEM},{ }^{*} P<0.001$ versus WT). (J) H\&E-stained sections of aortic root lesions from WT and $C \mathrm{cr} 2^{-1-}$ mice on day 28 after CAWS injection. Scale bars: $400 \mu \mathrm{m}$. Data in $\mathbf{G}$ and $\mathbf{H}$ are representative of 3 independent experiments. All $P$ values were calculated using unpaired 2-tailed Student's $t$ test.

homozygous mice (29). Equal numbers of $\mathrm{Ccr}^{2++} \mathrm{GFP}^{+}$and $\mathrm{Ccr} 2^{-1-}$ $\mathrm{RFP}^{+}$monocytes were isolated from the bone marrow (BM) of naive mice, mixed 1:1, and intravenously injected into WT mice along with i.p. CAWS injection. Twenty hours after adoptive transfer, tissues were harvested and analyzed for the presence of the $\mathrm{Ccr}^{+/+} \mathrm{GFP}^{+}$and $\mathrm{Ccr}^{-/-} \mathrm{RFP}^{+}$cells (Figure 2C). The ratio of $\mathrm{Ccr}^{+/+}$and $\mathrm{Ccr}^{-/-}$monocytes in the heart was skewed towards $\mathrm{Ccr} 2^{+/+}$monocytes at an approximately 3:1 ratio, while $\mathrm{Ccr} 2^{+/+}$ and $\mathrm{Ccr}^{-1-}$ monocytes were found in the blood at a 1:1 ratio (Figure 2, D and E). We also tracked the transferred WT GFP ${ }^{+}$ BM-derived monocytes (BMDMs) on day 7 after CAWS injection (Figure 2F). This revealed that the transferred monocytes not only migrated into the heart but also differentiated into CD11 $\mathrm{c}^{+}$ $\mathrm{MHCII}^{+} \mathrm{Mo}-\mathrm{DC}$ in the heart, which we found began to accumulate in the aortic root of the heart on day 7 (Figure 1B). GFP' cells in the heart showed increased expression of CD11c, MHCII, CD172 $\alpha$, and CD24, and decreased Ly6C expression, compared with the expression of these markers on these cells before trans- fer (Figure 2, G and $\mathrm{H}$ ), which is consistent with the transferred monocytes differentiating into Mo-DCs.

Given the importance of the CCL2/CCR2 pathway in early monocyte recruitment into the heart, we evaluated the vasculitis scores on day 28 in $\mathrm{Ccr}^{+/+}$and $\mathrm{Ccr}^{-/-}$mice. Notably, $\mathrm{Ccr} 2^{-/-}$ mice were totally protected from development of vasculitis (Figure 2, I and J). These results demonstrate that CAWS induced the CCR2-dependent recruitment of iMos into the heart in the early phase of cardiac inflammation and this process was necessary for induction of vasculitis.

Cardiac-resident macrophages are the main source of CCL2 in the early phase of vasculitis. Given the specific induction of CCL2 in the heart on day 1 after CAWS injection, we sought to identify the cellular source of CCL2 production using Ccl2-RFP fl/l $^{\prime / 2}$ mice, which report the cellular expression of the CCL2 protein (30). As shown by flow cytometric analysis of the entire heart obtained from Ccl2-RFP Pl/l $^{f /}$ mice, CCL2 was expressed by heart cells at 6 hours and became prominent at 18 hours after CAWS injection 
A
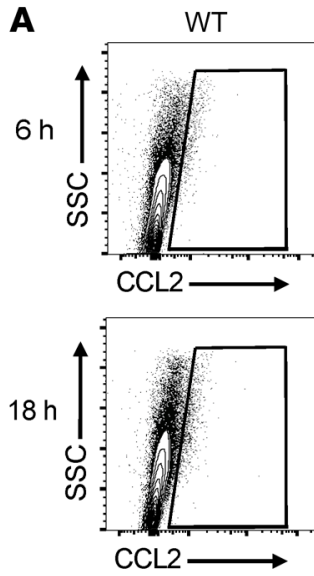

C

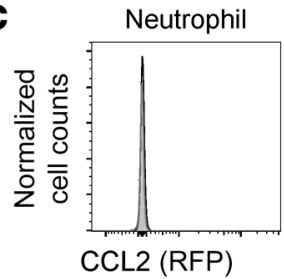

Ccl2-RFP
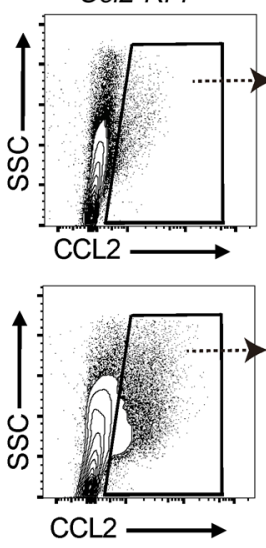

Live $\mathrm{CCL} 2^{+}$cells

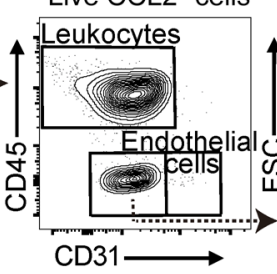

Leukocytes

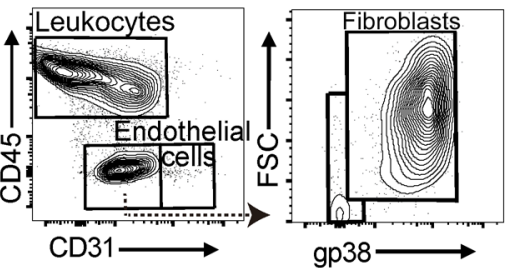

CD45-CD31+ cells

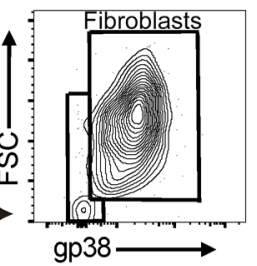

Ly6C ${ }^{\text {hi }}$ mono

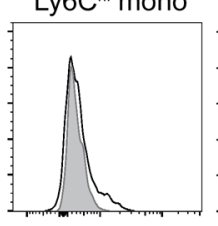

Mo-DC

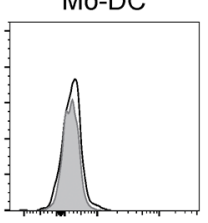

$\mathrm{cDC}$

Resident mac
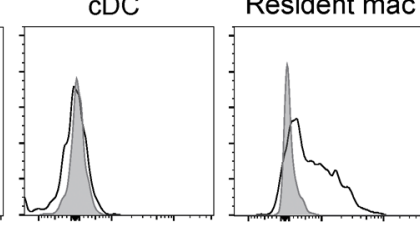

B \%

$\%$ Distribution of $\mathrm{CCL}^{+}$cells
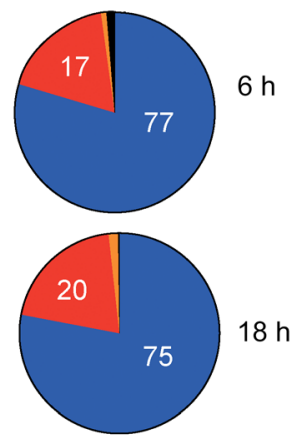

$\square$ Leukocytes

$\square$ Fibroblasts

$\square$ Endothelial cells

- Other stromal cells

Figure 3. Cardiac-resident macrophages are the main source of early CCL2 production. (A) Representative contour plots of RFP+ CCL2-expressing cells in live singlets derived from WT or Cc/2-RFPf/ffl reporter mouse hearts 6 hours and 18 hours after CAWS injection. One representative of 3 independent experiments. (B) Pie chart showing percentage distribution of $\mathrm{RFP}^{+}$cells for the indicated subpopulations in Cc/2-RFPf/f/ reporter mice heart 6 hours and 8 hours after CAWS injection. (C) Representative histograms of CCL2 expression in myeloid populations isolated from the hearts of Cc/2-RFPfl/fI reporter mice 1 day after CAWS injection ( $n=5$ mice). Individual leukocyte populations were immunophenotyped based on the gating strategy shown in Figure 1A. The RFP reporter was used to identify CCL2-producing cells.

(Figure 3A). To determine whether cardiac stromal cells or leukocytes are producing CCL2, we compared CCL2 expression in 4 cell subsets in the heart: cardiac leukocytes (CD45+CD31-), endothelial cells $\left(\mathrm{CD} 45^{-} \mathrm{CD} 31^{+}\right)$, fibroblasts $\left(\mathrm{CD} 45^{-} \mathrm{CD} 31^{-} \mathrm{gp} 38^{+}\right)$, and other stromal cells (CD45-CD31-gp38-), as previously described (25) (Figure 3A). Strikingly, leukocytes were the most abundant CCL2 producers at both 6 hours and 18 hours after CAWS injection, with minor production by fibroblasts (Figure 3B). Among the leukocyte population, $\mathrm{CD}^{+} 4^{+} \mathrm{MerTK}^{+}$cardiac macrophages preferentially expressed CCL2 on day 1 after CAWS injection compared with other cell types (Figure 3C). We further characterized cardiac macrophages in the entire heart on day 1 following CAWS injection by flow cytometry (Supplemental Figure 4). Cardiac macrophages uniformly expressed high levels of CD11b and F4/80, and lower levels of CD11c. A subpopulation of cardiac macrophages also expressed MHCII and Dectin-2, but cardiac macrophages did not express CCR 2 or Ly6C. These results suggest that cardiac-resident macrophages are the major source of early CCL2 production in the heart following CAWS injection.

CCL 2 is produced by $C D 11 b^{+}$Dectin-2 $2^{+}$resident cardiac macrophages in the adventitia of the aortic root and coronary vessels. To visualize the location of CCL2-producing cells, we histologically analyzed heart tissue from $C c l 2-R F P^{f / f l}$ mice on day 1 after CAWS injection and detected CCL2 protein expression in adventitia of the aortic root and coronary arteries (Figure 4, A-D). Although there was autofluorescence from cardiac myocytes, we could identify CCL2 protein expression associated with $C D 11 b^{+}$and Dectin- $2^{+}$cells, as well as occasional association with $\mathrm{CD} 11 \mathrm{c}^{+}$cells, but not with Ly $6 \mathrm{G}^{+}$neutrophils, confirming that CD11b ${ }^{+}$Dectin- $2^{+}$ cardiac macrophages were the main source of CCL2 on day 1 . To determine the pattern of CCL2 expression in macrophages in the aortic root area and in the myocardium, we injected CAWS into $C c l 2-R F P^{f / f}$ reporter mice and harvested the heart on day 1 for immunofluorescence analysis following staining for CD11b. CCL2 was preferentially produced by $\mathrm{CD} 11 \mathrm{~b}^{+}$cells in the aortic root area compared with the myocardium (Supplemental Figure 5). Although we detected a number of CD11 $\mathrm{b}^{+}$cells in myocardium on day 1, most of the CD11 $\mathrm{b}^{+}$cells did not express CCL2.

CAWS components are delivered to the adventitia of the aortic root. Our results suggest that CCL2 was expressed exclusively in the heart 1 day following CAWS injection, and that the main source of CCL2 was cardiac macrophages. We next examined why and how cardiac macrophages are activated following CAWS injection into the peritoneal cavity. We first hypothesized that Dectin-2 expression might be higher in cardiac macrophages compared with macrophages from different organs, making them more sensitive to CAWS stimulation. However, quantitative real-time PCR (qPCR) analysis of sorted macrophages isolated from various organs of naive mice indicated that Dectin-2 expression was not higher in cardiac macrophages compared to other organs (data not shown). Then we asked if CAWS is delivered to the site of future inflammation in the heart and thereby directly inducing macrophage activation. To this end, we performed immunofluorescent staining for CAWS using an antibody that reacts with proteins in the CAWS extract (Figure 5, A-C). Intriguingly, Candida components (green) were detected on day 1 in the aortic root and were associated with 
A

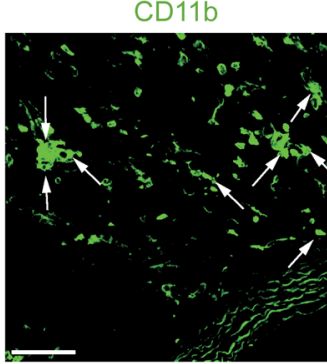

B

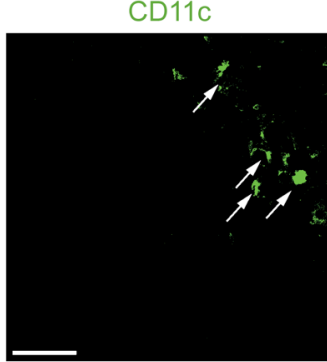

C

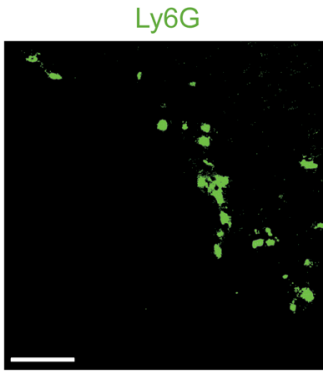

D
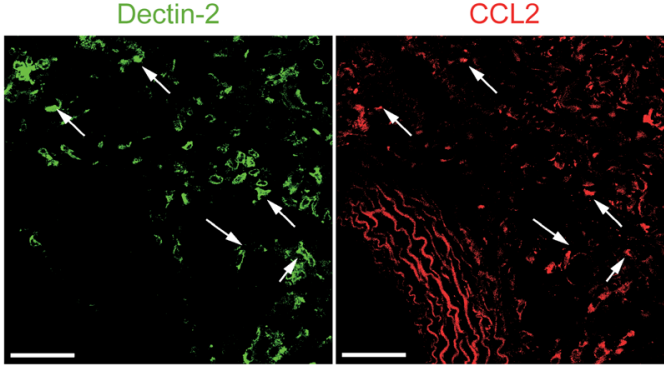

CCL2

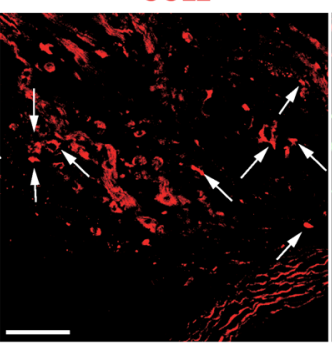

CCL2

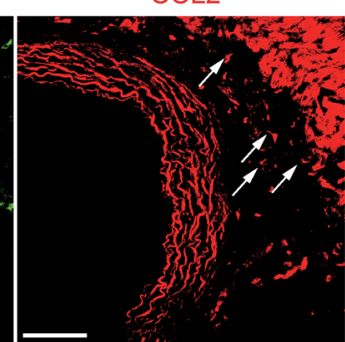

CCL2
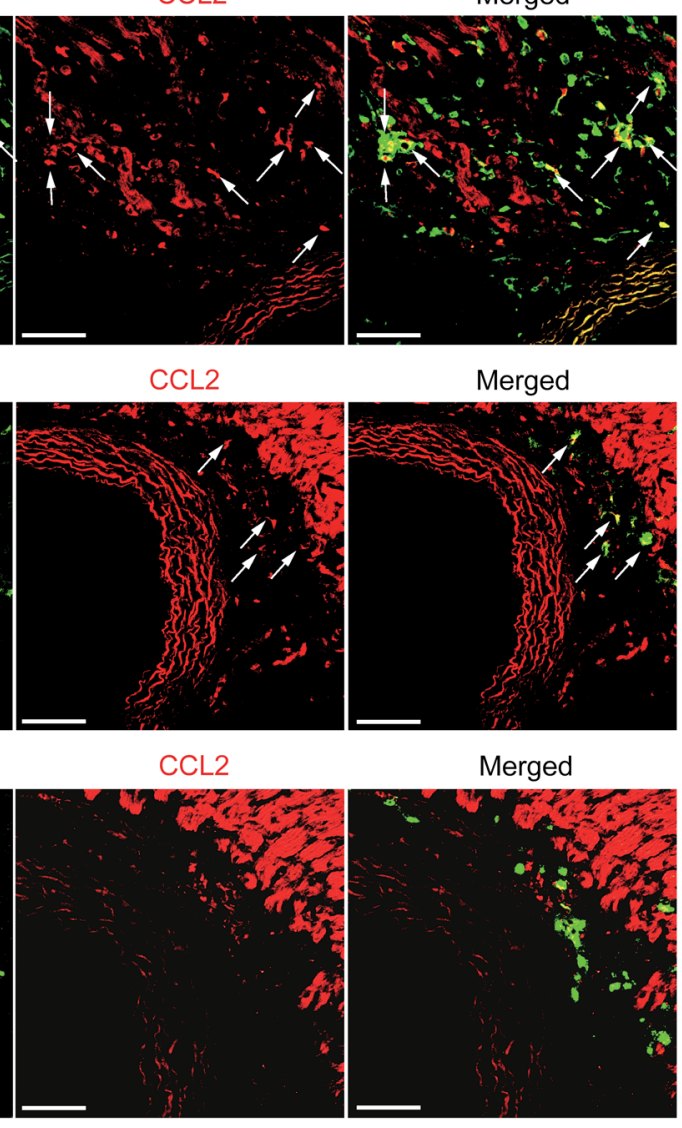

Merged

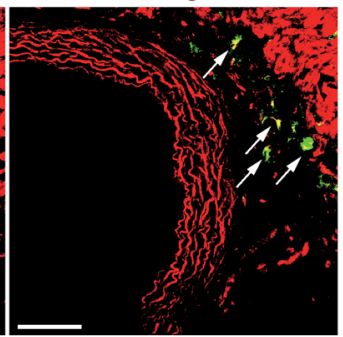

Merged

Merged

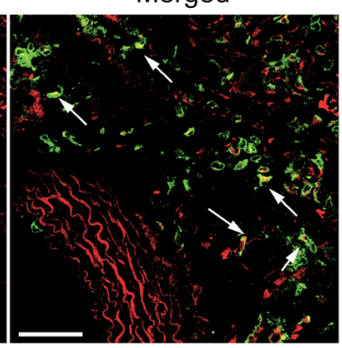

Figure 4. CCL2 is produced by CD11b+Dectin-2+ resident cardiac macrophages around the aorta. (A-D) Heart tissue of CCl2-RFPfl/fl reporter mice on day 1 after CAWS injection was stained for CD11b (A), CD11C (B), Ly6C (C), and Dectin-2 (D) (all green) and analyzed by confocal microscopy. Arrows indicate colocalization with CCL2 (red). Scale bars: $40 \mu \mathrm{m}$.

phages in CCL2 production after CAWS. Because cardiac macrophages express F4/80 (Supplemental Figure 4 ), we isolated $\mathrm{F} 4 / 80^{+}$cells from singlecell suspensions of naive hearts using magneticactivated cell sorting and then stimulated these cells with CAWS. CCL2 production was induced by CAWS in $\mathrm{F} 4 / 80^{+}$cardiac macrophages derived from WT mice but not from Dectin-2-/- mice (Figure 6A). Furthermore, CCL2 mRNA levels in heart tissue on day 1 following CAWS injection was markedly reduced in Dectin-2/- mice compared with WT mice (Figure 6B). Because cardiac fibroblasts also produced CCL2 after CAWS injection (Figure 3B), we determined whether CAWS was also capable of directly stimulating CCL2 production in primary cardiac fibroblasts in vitro. Cardiac fibroblasts did not produce CCL2 mRNA or protein in response to CAWS stimulation in vitro; however, IL-1 $\beta$, TNF- $\alpha$, and LPS were able to induce CCL2 mRNA and protein in cardiac fibroblasts (Supplemental Figure 6). Although cardiac fibroblasts also contribute to CCL2 production in the heart, our results suggest they are not responding directly to CAWS but are presumably responding to other endogenous mediators released in the heart tissue in response to CAWS.

In addition to the CLRs Dectin-1 and Dectin-2, TLR2 and TLR4 have been reported to recognize Candida PAMPs and contribute to Candida immunity (31). To determine which PRR is crucial for CAWS recognition and vasculitis induction,

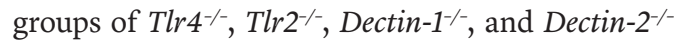
mice were injected with CAWS and vasculitis was assessed histologically. Dectin-2 $2^{-/-}$mice were completely protected from vasculitis, while $\mathrm{Tlr}^{-/}$, Tlr2 ${ }^{-/}$, and Dectin-1/-- mice were only partially protected from vasculitis compared with WT mice (Figure 6, C and D). These results indicate that Dectin-2, which recognizes the $\alpha$-mannans on fungal hyphae, is a critical PRR for CAWS-induced vasculitis. Dectin-2 signals through CARD9 and Syk, resulting in NF- $\kappa$ B activation (23). The $\mathrm{FcR} \gamma$ chain is required for the surface expression and signaling of Dectin-2. Consistent with our Dectin-2-/

$\mathrm{CD}_{11 \mathrm{~b}^{+}}$cells (red) (Figure 5B). In contrast, Candida particles were barely detectable in other organs, such as the lung, spleen, liver,

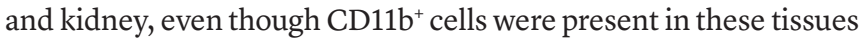
(Figure 5C). Notably, Candida particles were not detected in the myocardium on day 1 following CAWS injection (Figure 5, A and C). These results suggest that the preferential deposition of CAWS antigen in the adventitia of the aortic root, which is rich in vasa vasorum and includes the ostium of the coronary arteries, determines the cardiac vessel-specific inflammation in this model.

Dectin-2 is required for CAWS-induced CCL2 production and development of arteritis. Given the role of Dectin-2 in recognition of mannans and the expression of this PRR on cardiac macrophages, we sought to define the role of Dectin- $2^{+}$cardiac macro- results, $\mathrm{Fc} \gamma \mathrm{R}^{-/-}$and $\mathrm{Card} 9^{-/-}$mice were also completely protected from CAWS-induced vasculitis (Figure 6E). As a Dectin- $2^{f / f l}$ strain was not available, we alternatively assessed the contribution of Syk-dependent pathways directly in CD11 $\mathrm{c}^{+}$cells during the development of vasculitis. We crossed $S y k^{f / f l}$ mice (32) with CD11c-Cre mice (33) to generate a $C D 11 \mathrm{c} \Delta$ Syk strain that specifically lacks Syk in CD11c-expressing cells. Notably, CD11c $\Delta$ Syk mice were completely protected from vasculitis compared with littermate controls $\left(S y k^{f l f l}\right)$ (Figure 6F). One day after CAWS injection, CCL2 mRNA transcripts in the heart were markedly lower in CD11c $\Delta$ Syk mice compared with control mice, implying a contribution of heart $\mathrm{CD} 11 \mathrm{c}^{+}$cells (possibly CD11 $\mathrm{c}^{+}$cardiac macrophages) in the production of CCL2 in response to CAWS (Figure 6G). 
A

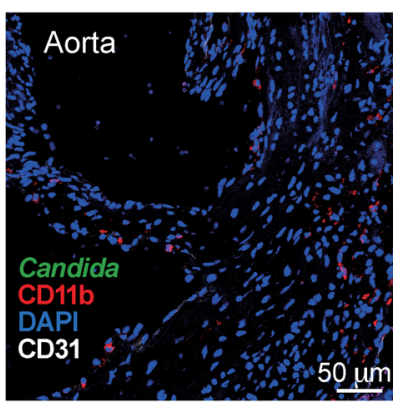

B

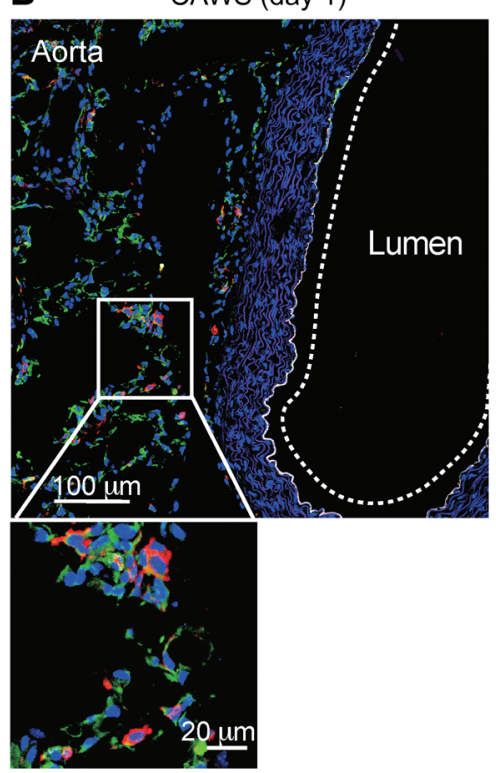

C

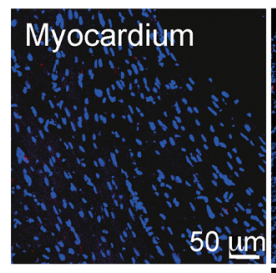

CAWS (day 1)
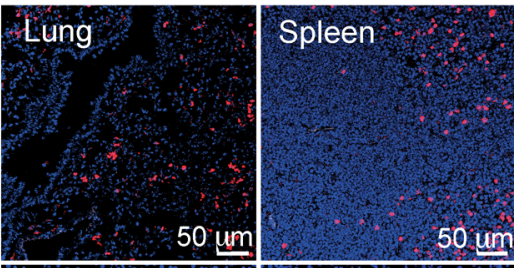

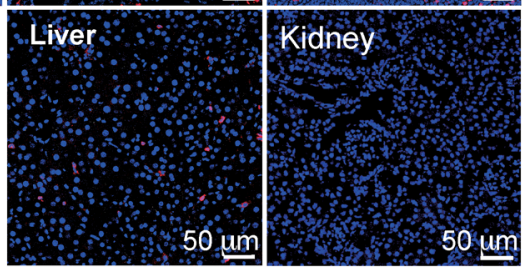

Figure 5. CAWS components are delivered to the aortic root area on day 1 after CAWS injection. Each organ was isolated from naive (A) or CAWS-injected WT mice on day 1 (B and C) and stained with anti-Candida albicans (green), anti-CD11b (red), and anti-CD31 (white). Representative images of 6 mice from 3 experiments are shown.

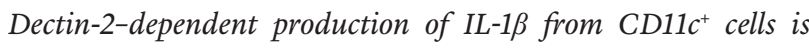
required for CAWS-induced arteritis. To examine the key cytokines in CAWS-induced arteritis, we performed qPCR analysis on RNA isolated from heart tissue on day 28 after CAWS treatment. IL-1 $\beta$ was highly expressed in the heart following CAWS treatment compared with other proinflammatory cytokines, including IL-6, TNF- $\alpha$, and IL-1 $\alpha$ (Figure 7A). We then examined the role of $\mathrm{IL}-1 \alpha / \beta$ in the development of arteritis using $I L 1 a^{-/-} I L 1 b^{-/-}$mice. Remarkably, $I L 1 a^{--} I L 1 b^{-/}$mice were completely resistant to CAWS-induced arteritis (Figure 7B). Next, we generated BM chimeric (BMC) mice to address whether IL- $1 \alpha / \beta$ are required in hematopoietic cells or radioresistant resident cells for arteritis development. After BM engraftment, chimeric mice were injected with CAWS and vasculitis scores were evaluated on day 28 (Figure 7C). BMC mice that had WT BM transplanted into $(\rightarrow)$ WT recipient mice were susceptible to arteritis. In contrast, $I L 1 a^{-/} \mathrm{IL}_{1} b^{-/} \rightarrow$ $I L 1 a^{-/} I L 1 b^{-/-}$controls were resistant to arteritis, as were $I L 1 a^{-1-}$ $I L 1 b^{-/} \rightarrow$ WT BMC. However, WT $\rightarrow I L 1 a^{-/} I L 1 b^{-/-}$BMC mice developed arteritis comparable to WT $\rightarrow$ WT controls (Figure 7D). These results demonstrate that IL- $1 \alpha / \beta$ expression in radiosensitive cells is necessary and sufficient for arteritis, whereas IL- $1 \alpha / \beta$ expression in radioresistant cells does not contribute to the development of arteritis.

To further identify the cellular source of IL-1 $1 \beta$, we utilized pIL1-DsRed-transgenic mice expressing the Discosoma red fluorescent protein (DsRed) gene under the control of the IL-1 $1 \beta$ promoter (34). We analyzed DsRed-expressing leukocyte subsets in the heart during the course of arteritis using the gating strategy described in Supplemental Figure 7. Distribution of IL-1 $\beta$-producing DsRed ${ }^{+}$cells showed that following CAWS injection, IL-1 $\beta$ was actively transcribed in neutrophils on day 1 (early phase), followed by Mo-DCs on day 7 (middle phase), and then was abun- dantly produced in neutrophils on day 28 (late phase) (Figure 7E). To determine if Dectin-2 was necessary for IL-1 $\beta$ production in the heart, we evaluated IL-1 $\beta$ expression in WT and Dectin-2hearts on days 7 and 28 after CAWS injection. IL-1 $1 \beta$ mRNA levels were markedly reduced in Dectin-2- hearts compared with WT hearts on days 7 and 28 after CAWS injection (Figure 7F). Similarly, IL-1 $\beta$ mRNA levels in CD11c $\Delta$ Syk hearts were significantly reduced compared with control hearts on day 7 and 28 after CAWS injection (Figure 7G). These results indicate that both Dectin-2 and Syk signaling in CD11 $\mathrm{c}^{+}$cells is required for IL-1 $\beta$ expression in the heart after CAWS injection.

CAWS activates the NLRP3 inflammasome and promotes IL-1 $\beta$ production via Dectin-2. Translocation of cytoplasmic NF- $\mathrm{\kappa B}$ p 65 to the nucleus is a key step in the activation of the NF- $\mathrm{kB}$ pathway. To determine whether Dectin-2 was required for CAWS-induced acti-

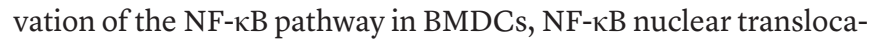
tion was assessed by immunofluorescent staining. Sixty minutes following stimulation of BMDCs with CAWS, or LPS as a positive control, NF- $\mathrm{kB}$ p65 (red) was detected in the cell nucleus (blue). CAWS induced NF- $\mathrm{\kappa B}$ p 65 translocation from the cytoplasm to the nucleus in WT BMDCs, but this translocation was largely absent in Dectin-2-/ BMDCs (Figure 8A). NF- $\mathrm{kB}$ nuclear translocation was assessed and quantified as the percentage of cells that had p65-positive nuclei versus total cells (Figure 8B). Similar results were obtained from Dectin-2-- Mo-DCs (data not shown). Recognition of microbial signals by inflammasome proteins, such as NLRP3 and AIM2, triggers assembly of the inflammasome. Upon formation of the inflammasome complex, procaspase-1 is cleaved into an active cysteine protease, which further cleaves IL-1 $1 \beta$ into mature forms (35). To test the functional role of inflammasome activation in IL-1 $\beta$ production in CAWS-induced arteritis, groups of Aim2 $2^{--}$, Nlrp3 $3^{--}$, or Caspase-1-/ mice were injected with CAWS 


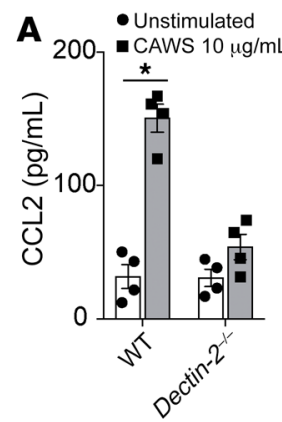

E

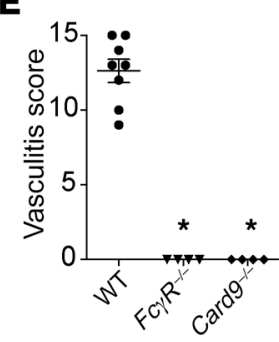

B

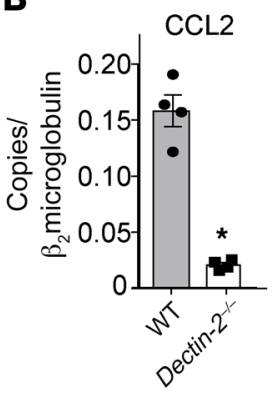

$\mathbf{F}$

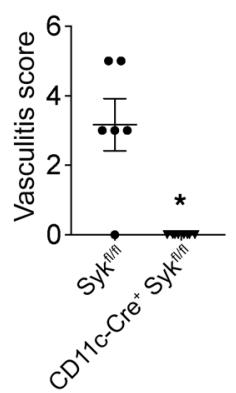

C
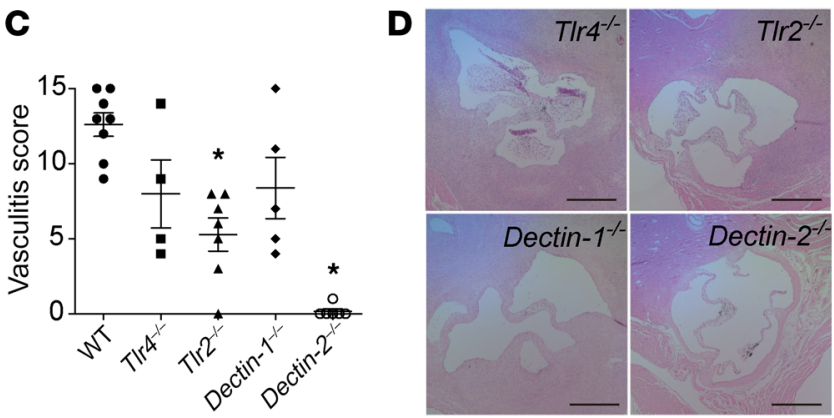

G CCL2

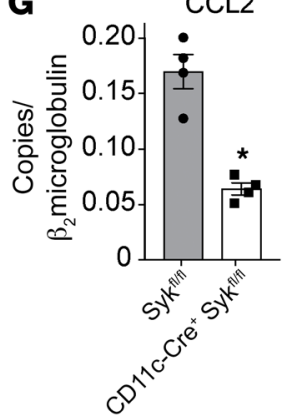

Figure 6. Dectin-2 is required for CAWS-induced CCL2 production and the development of vasculitis. (A) F4/80+ cells isolated from the heart were stimulated with CAWS $(10 \mu \mathrm{g} / \mathrm{mL}$ ) for 18 hours and CCL2 protein in the culture supernatant was measured by ELISA (mean \pm SEM from 2 experiments; ${ }^{*} P=0.001$ versus unstimulated). (B) Wild-type and Dectin- $2^{-/-}$mice were injected with CAWS, and 1 day later hearts were harvested and assessed for

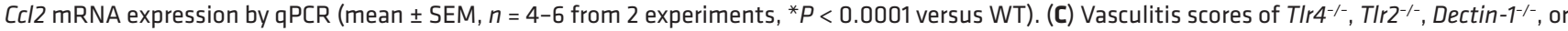
Dectin-2/- mice on day 28 after CAWS injection (mean \pm SEM, $n=4-8$ mice per group, ${ }^{*} P<0.001$ versus WT). (D) H\&E-stained aortic root lesions isolated

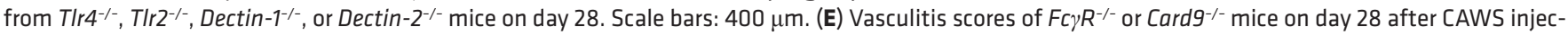
tion (mean $\pm \mathrm{SEM},{ }^{*} P<0.0001$ versus WT). (F) Vasculitis scores of CD11c $\Delta$ Syk mice or control mice (Syk $k^{f l / f}$ ) on day 28 after CAWS injection (mean \pm SEM, ${ }^{*} P<0.0001$ versus Syk $\left.{ }^{f / f}\right)$. (C) CD11c $\Delta$ Syk mice or control mice were injected with CAWS, and 1 day later, heart tissues were harvested and assessed for $C c / 2$ RNA expression by qPCR (mean $\pm \mathrm{SEM}, n=4$ mice per group, ${ }^{*} P<0.001$ versus $\left.S y k^{f / / f l}\right)$. $P$ values were calculated using unpaired 2 -tailed Student's $t$ test (A, $\mathbf{B}, \mathbf{F}$, and $\mathbf{G}$ ) or 1-way ANOVA with Dunnett's post hoc test (C and $\mathbf{E}$ ).

and vasculitis was histologically evaluated. Deficiency of Nlrp3, Caspase -1 , and $I L 1 a / b$ protected mice from arteritis, whereas mice deficient in Aim2 developed full arteritis (Figure 8, C and D).

NLRP3 inflammasome activation requires 2 signals: signal 1, NF- $\mathrm{KB}$ activation inducing the transcription of NLRP3 and proIL-1 $\beta$; and signal 2, NLRP3 inflammasome activation inducing the cleavage of pro-IL-1 $\beta$ into bioactive IL-1 $\beta$ (36). To investigate whether CAWS directly induced IL-1 $\beta$ production, BMDCs from Aim 2- $2^{--} N L R P 3^{--}$, or Caspase- $1^{-/-}$mice were stimulated with CAWS with or without the signal 1 inducer LPS or the signal 2 inducer monosodium urate crystals (MUCs) for 18 hours, and IL-1 $\beta$ release was determined. In combination with either LPS or MUCs, CAWS synergistically promoted IL- $1 \beta$ secretion in an NLRP3- and caspase-1-dependent manner (Figure 8E). These results indicate that CAWS induces NLRP3/caspase-1-dependent IL-1 $\beta$ maturation in DCs, which is required for CAWS-induced arteritis. Further, because CAWS synergized with both signal 1 and signal 2 inducers, this suggests that CAWS can act as both a signal 1 and signal 2 inducer.

BMDCs from Dectin-1-/, Dectin-2-/, or Card9 ${ }^{-/-}$mice were also stimulated with CAWS with or without the signal 2 stimulator MUCs for 18 hours, and IL- $1 \beta$ release was determined. Secretion of IL-1 $\beta$ from CAWS-stimulated BMDCs was not observed in Dectin-2- and Card $^{-/}$cells (Figure 8F). Similar results were obtained from Dectin-2-^ Mo-DCs (data not shown). These results demonstrate that CAWS directly acts as a signal 1 and signal 2 via Dectin- 2 activation to induce the production of IL-1 $1 \beta$ in Mo-DCs, which are the major IL-1 $1 \beta$ producers on day 7 .

Role of $I L-1 \beta$ in chemokine and adhesion molecule expression. MyD88 is required for most TLR signaling as well as for IL-1 receptor 1 (IL-1R1) signaling. To investigate whether MyD88 signaling in radiosensitive cells or radioresistant cells was required for CAWS-induced vasculitis, we generated BMC mice using WT and $\mathrm{MyD} 88^{--}$mice (Figure 9A). $\mathrm{MyD} 88^{-/} \rightarrow \mathrm{MyD} 88^{-/-}$controls were resistant to arteritis, as were WT $\rightarrow M y D 88^{-/}$BMC. However, $\mathrm{MyD} 88^{--} \rightarrow$ WT BMC developed arteritis comparable to WT $\rightarrow$ WT controls (Figure 9, B and C). These results indicate that IL-1R1/MyD88 signaling in radioresistant cells, such as endothelial cells, is necessary for arteritis, whereas TLRs or IL-1R1/MyD88 signaling in hematopoietic cells does not contribute to the development of CAWS-induced arteritis.

To determine whether IL- $1 \beta$ was an important mediator of chemokine and adhesion molecule induction in the model, we stimulated mouse aortic endothelial cells (MAECs) with IL-1 $\beta$ and measured chemokine and adhesion molecule expression by ELISA and flow cytometry, respectively. In MAECs, IL-1 $\beta$ stimulated the production of chemokine proteins mediating monocyte and neutrophil recruitment, such as CCL2 and CXCL1, respectively (Fig- 

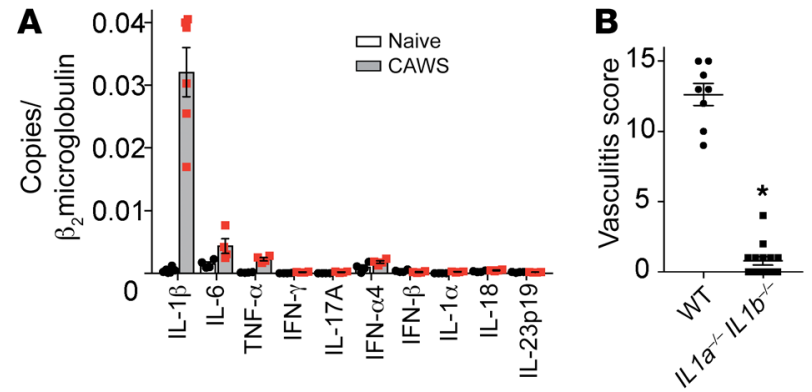

C

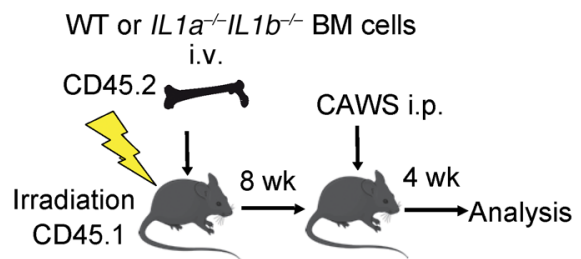

D

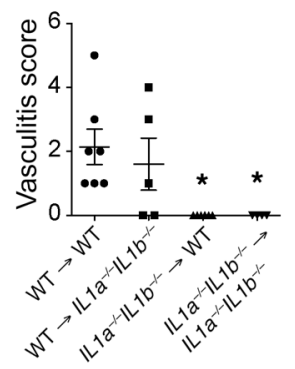

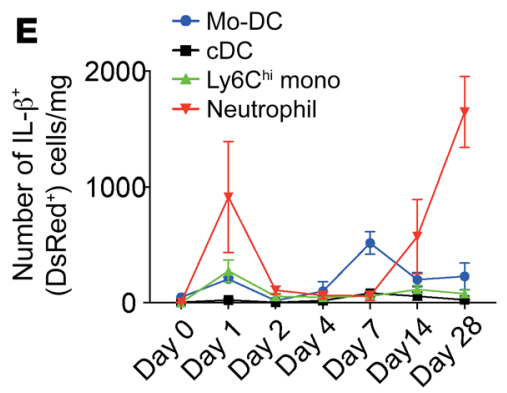

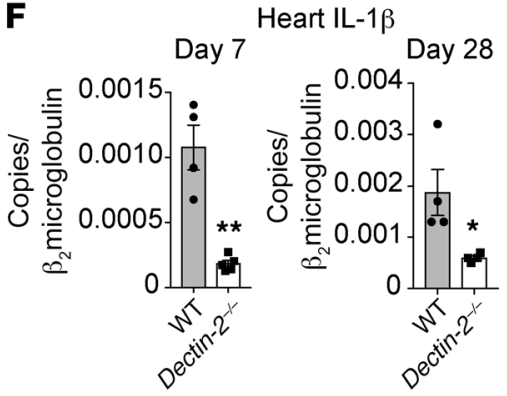

G

Heart IL-1 $\beta$

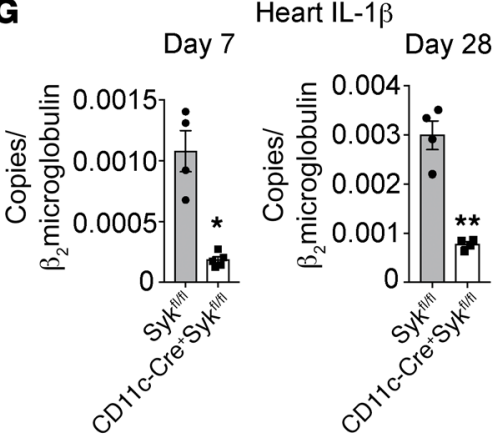

Figure 7. Dectin-2-dependent production of IL-1 $\beta$ from CD11c cells is required for CAWS-induced vasculitis. (A) Heart tissue from WT mice was harvested 28 days after initial CAWS injection and assessed for cytokine expression by qPCR $(n=4-5$ per group, mean \pm SEM). (B) Vasculitis scores of $I L 1 a^{-/-} I L 1 b^{-/-}$mice or WT mice 28 days after CAWS injection (mean $\pm \mathrm{SEM},{ }^{*} P<0.0001$ versus WT). (C) Schematic of BMC mice generation using WT and

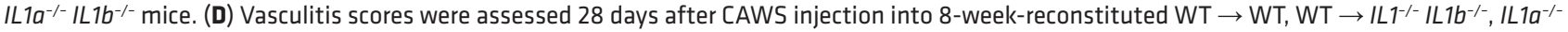
$\mathrm{IL} 1 b^{-/-} \rightarrow I L 1 a^{-1-} I L 1 b^{-1-}$, and IL $1 a^{-1-} I L 1 b^{-1-} \rightarrow$ WT BMC mice (mean \pm SEM, ${ }^{*} P<0.01$ versus WT $\rightarrow$ WT). (E) Kinetics of IL-1 $\beta^{+}$(DsRed ${ }^{+}$) cell numbers of the indicated immune cell subset per mg of heart tissue determined by flow cytometric analysis on day 0, 1, 2, 4, 7, 14, or 28 after CAWS injection of pll1-DsRed mice (mean \pm SEM, $n=3$ per time point). (F) Dectin-2-/- or WT mice were injected with CAWS and 7 or 28 day later hearts were harvested and assessed for IL-1 $\beta$ expression by qPCR (mean \pm SEM, $n=4-5$ per group). (C) CD11c $\Delta$ Syk mice or control mice were injected with CAWS and 7 or 28 day later hearts were harvested and assessed for IL-1 $\beta$ expression by qPCR (F and $\mathbf{G}$; mean $\pm \mathrm{SEM},{ }^{*} P<0.05$, ${ }^{*} P<0.001$ versus Syk ${ }^{f \mid / f f}$ ). $P$ values were calculated using unpaired 2-tailed Student's $t$ test (B, $\mathbf{F}$, and $\mathbf{G}$ ) or 1-way ANOVA with Dunnett's post hoc test (D).

ure 9D). The cell surface expression of ICAM-1, VCAM-1, E-selectin, and P-selectin was also elevated in IL-1 $\beta$-stimulated MAECs (Figure 9E). Similarly, IL-1 $\beta$ also stimulated monocyte- and neutrophil-attracting chemokine production from primary cardiac fibroblasts (Supplemental Figure 8). We also performed immunofluorescent staining of ICAM-1, VCAM-1, and CD31 on heart tissue isolated on day 28 after CAWS injection (Supplemental Figure 9). ICAM-1 was stained within the adventitial vessels, whereas VCAM-1 was stained in the media of the aortic root. In addition to endothelial cells, mouse VCAM-1 is known to be expressed by hematopoietic cells, such as macrophages, granulocytes, and $\mathrm{T}$ cells. It is presumed that in this vasculitis model, IL-1 $\beta$ promotes ICAM-1 expression in the adventitial vasa vasorum, driving adhesion and recruitment of inflammatory cells into the aortic root area.

To determine the role of Dectin-2 and IL-1 $\beta$ in chemokine induction in cardiac tissue in vivo, we measured the levels of chemokine mRNA by qPCR in $I L 1 a^{-/-} \mathrm{ILIb}^{-/-}$and Dectin-2-/- mice on day 28 following CAWS injection. We observed markedly reduced levels of mRNA for monocyte-attracting CCR2 chemokine ligands, such as CCL2, CCL7, and CCL12, in $\mathrm{LL}_{1 \mathrm{a}^{-/}} \mathrm{IL} 1 \mathrm{~b}^{-/-}$ and Dectin-2 ${ }^{-/-}$mice compared with WT mice. In addition, levels of mRNA for the neutrophil-attracting CXCR2 chemokine ligands CXCL1, CXCL5, and CXCL7 were also decreased in $I L 1 a^{-/-} I L 1 b^{-/-}$ and Dectin- $2^{-/-}$mice (Figure 9F). Accordingly, levels of mRNA for
CCR2 as well as CCR1 and CXCR2 (murine neutrophil-attracting chemokine receptors) were reduced in the hearts of $I L 1 a^{-/} I L 1 b^{-/-}$ and Dectin-2-/- mice on day 28 following CAWS injection compared with WT mice (Figure 9G). These data suggest that chemokines and chemokine receptors important for iMo and neutrophil recruitment are expressed in the heart in the later phase of arteritis in a Dectin-2- and IL- $1 \alpha / \beta$-dependent manner.

\section{Discussion}

CAWS is a mannoprotein- $\beta$-glucan complex produced and secreted by Candida albicans that induces cardiac arteritis resembling human KD when injected intraperitoneally into mice. CAWS is noninfectious since it does not contain live fungal cells, and is considered a PAMP that activates the innate immune response (37). Here we found that CAWS directly stimulated $\mathrm{F} 4 / 80^{+}$cardiac tissue-resident macrophages (TRMs) in the adventitia of the aortic root and coronary arteries to produce CCL2 in a Dectin-2-dependent manner. CCL2 induced iMo recruitment into the adventitia of the aortic root and coronary arteries of the heart where they differentiated into Mo-DCs, amplifying vessel inflammation by producing IL-1 $\beta$, which resulted in neutrophil- and monocyteattracting chemokine release from endothelial cells and fibroblasts leading to massive inflammatory cell infiltration (Supplemental Figure 10). Strikingly, the CCR2 ligand CCL2 was exclusively 

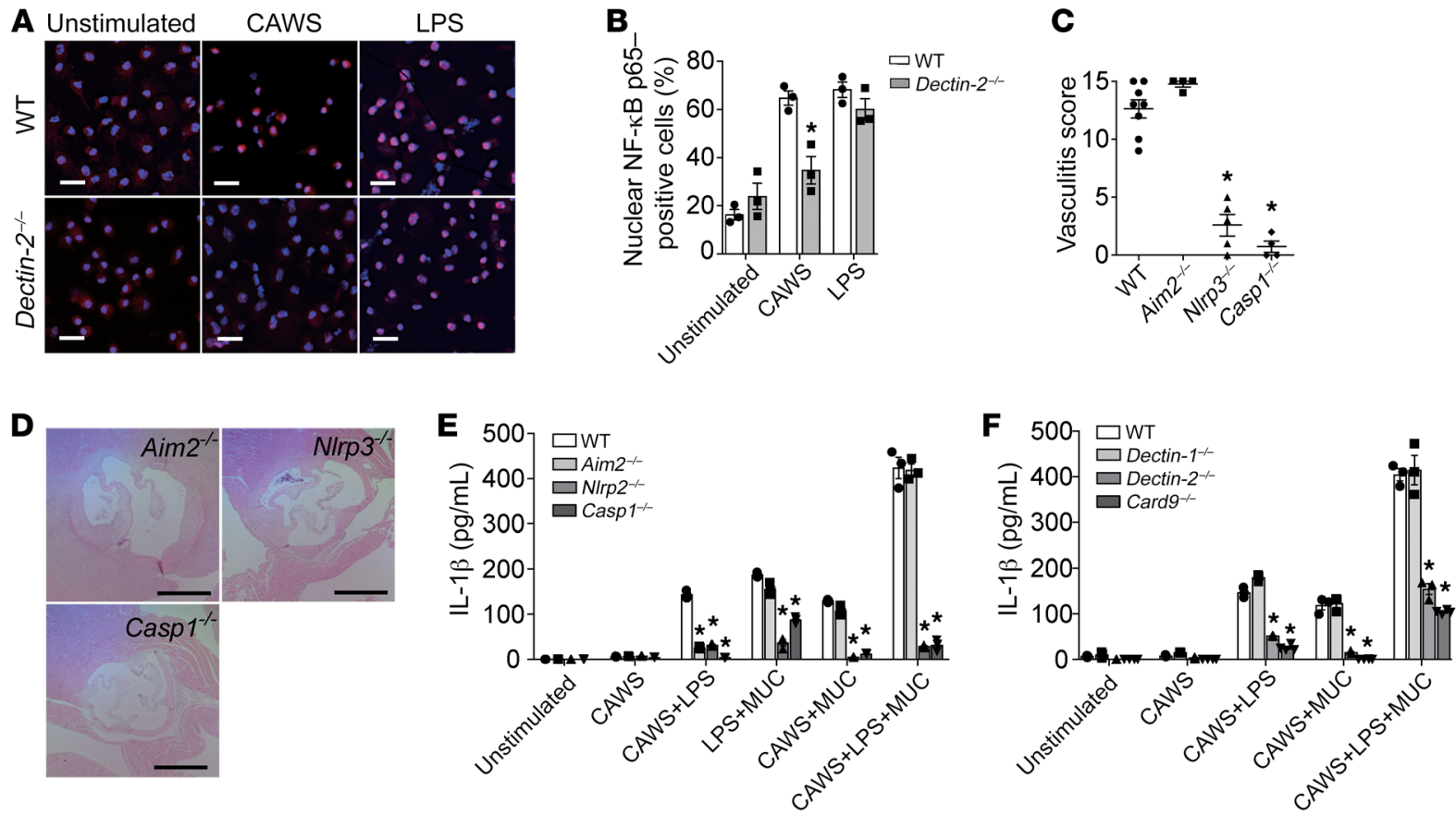

Figure 8. CAWS activates the NLRP3 inflammasome and promotes IL-13 production via Dectin-2. (A) Representative immunofluorescent staining showing NF-кB p65 (red) nuclear translocation in WT and Dectin-2-/- BMDMs stimulated for 60 minutes with CAWS (10 $\mu \mathrm{g} / \mathrm{mL})$ or LPS $(1 \mu \mathrm{g} / \mathrm{mL})$. Cell nuclei were detected by DAPI (blue). Similar results are obtained from 3 independent experiments. Scale bars: $20 \mu \mathrm{m}$. (B) Quantitation of NF- $\mathrm{KB}$ p65 nuclear translocation in the indicated groups. Results are expressed as the percentage of cells with NF- $\kappa B$ p65-positive nuclei versus total cells. Similar results were obtained from 3 independent experiments (mean $\pm \mathrm{SEM}, n=3$ per group, ${ }^{*} P<0.01$ versus WT). (C) Vasculitis scores of WT, Aim2 $2^{-1-}$, NLRP3 ${ }^{-1 /}$, or Caspase $-1^{-1-}$ mice on day 28 after CAWS injection (mean \pm SEM, $n=4-8$ mice per group, ${ }^{*} P<0.0001$ versus WT). (D) H\&E-stained sections of aortic root

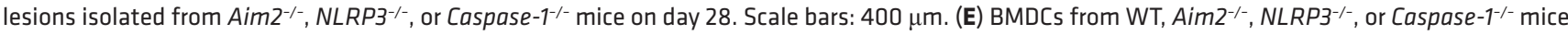
were stimulated with CAWS with or without inflammasome stimulators (LPS and MUCs) for 18 hours, and IL-1 $\beta$ release was assessed by ELISA (mean \pm SEM, $n=3$ per group, ${ }^{*} P<0.0001$ versus WT). (F) BMDCs from Dectin- $1^{-1-}$, Dectin- $2^{-1-}$, or Card $9^{-/-}$mice were stimulated with CAWS with or without inflammasome stimulators (LPS and MUC) for 18 hours, and IL-1 $\beta$ release was assessed by ELISA (mean \pm SEM, $n=3-4$ per group, ${ }^{*} P<0.0001$ versus WT). $P$ values were calculated using unpaired 2-tailed Student's $t$ test (B) or 1-way ANOVA with Dunnett's post hoc test (C, E, and $\mathbf{F})$.

expressed in the aortic root area after CAWS injection, suggesting that tissue-specific expression of CCL2 directs vascular inflammation in this model.

TRMs consist of heterogeneous populations of macrophages distributed throughout the body that facilitate homeostasis and immunosurveillance (38). Tissue heterogeneity of TRMs is programmed by local factors derived from tissue environments, which induce selective genetic and epigenetic programs that define the phenotype and function of TRMs $(39,40)$. In fact, it has been demonstrated that TRMs from different tissues, such as the heart, brain, lung, and peritoneal cavity, have a different transcriptome, and that the CCR2 ligand CCL2 is expressed at 200-fold higher levels in cardiac macrophages compared with other TRMs (41). In addition, TRMs are equipped with a variety of PRRs that sense microorganisms and produce cytokines and chemokines to recruit and activate immune cells (42). Here we have found that cardiac TRMs play an important role in initiating vasculitis by sensing CAWS via Dectin-2 and releasing CCL2. Ccr2 ${ }^{-/-}$mice have been shown to be protected against CAWS-induced vasculitis (43), which is consistent with our findings. Although this prior study found that iMos were mobilized from the BM in a Ccr2-dependent manner, they did not demonstrate that CCR2 also controls iMo entry into the heart where they contribute to vessel inflammation. Although we found that CCL2 was exclusively induced in the heart after CAWS injection in vivo, CAWS was able to induce CCL2 in vitro in TRMs isolated from other organs, and Dectin-2 RNA was detected in the spleen and lung as well as the heart at baseline (data not shown). We found that CAWS components are delivered to the aortic root area of the heart on day 1 following CAWS injection, whereas they were barely detectable in other organs and in the myocardium of the heart. The preferential deposition of CAWS PAMPS may explain the mechanism of cardiac-specific induction of CCL2 in this model.

Consistent with our data, Dectin-2 has been shown to be required for CAWS-induced IL-1 $\beta$ production by BMDCs, and that CAWS stimulation activates Syk, MAPKs, and NF- $\kappa$ B in BMDCs in a Dectin-2-dependent manner in vitro (20). We now demonstrate that Dectin-2 is required for CAWS-induced vasculitis in vivo and IL-1 $\beta$ production in vitro. We also found that (a) IL-1 $\beta$ was markedly induced in the inflamed heart; (b) production of IL- $1 \alpha / \beta$ from hematopoietic cells was required for CAWS-induced vasculitis; and (c) MyD88, which mediates IL-1R1 signaling, was required in stromal cells for CAWS-induced vasculitis. Among the myeloid cell populations, IL-1 $\beta$ was mainly produced by Mo-DCs in the middle phase of vasculitis (day 7), whereas neutrophils were the main IL-1 $\beta$ producers in the early (day 1 ) and late phases (day 28). In addition, we demonstrated that CD11 $\mathrm{c}^{+}$cell-specific deletion of the Dectin-2 signaling molecule Syk attenuated both 

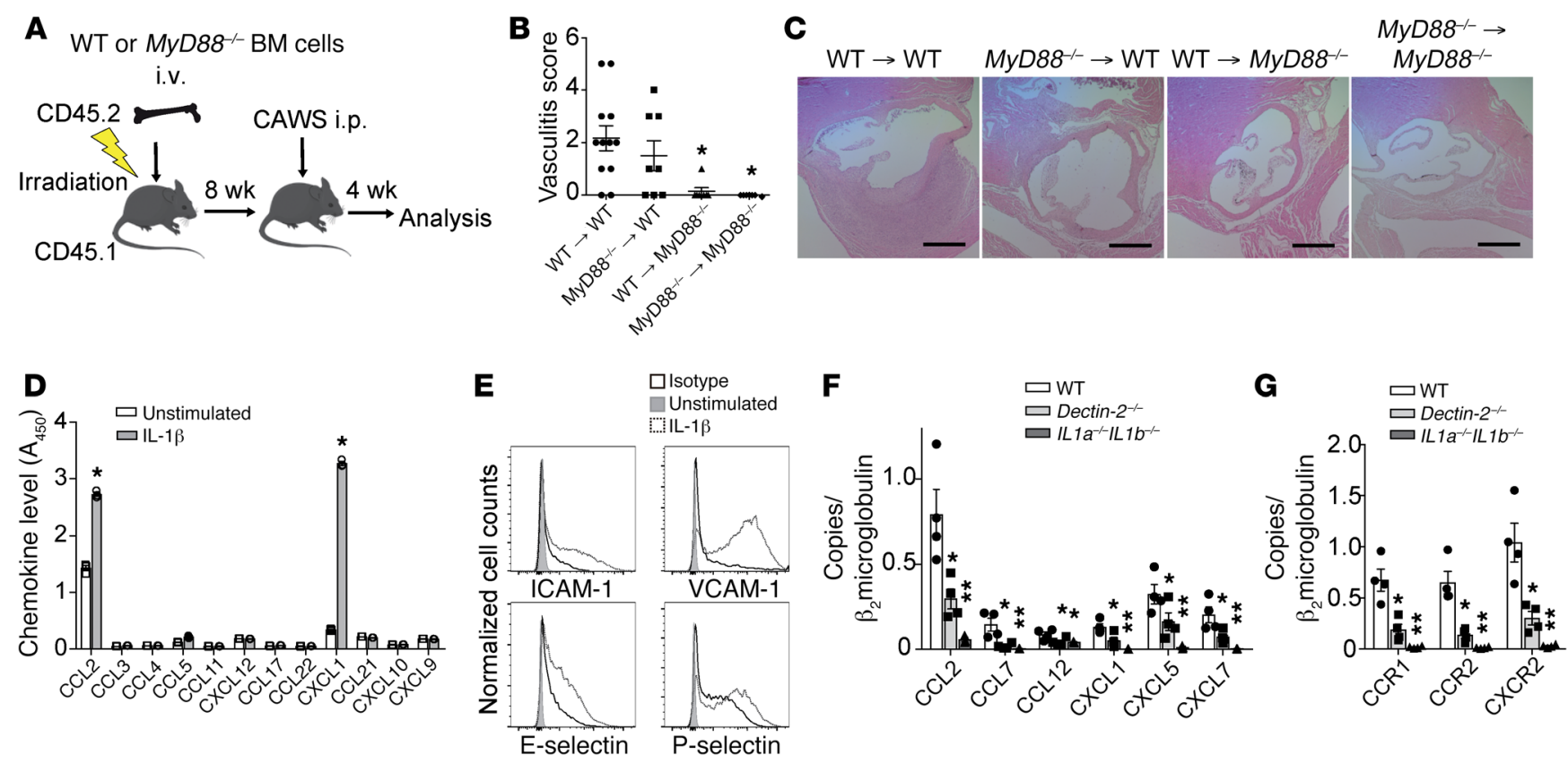

Figure 9. IL-1B/MyD88 signaling in heart stromal cells is required for chemokine and adhesion molecule induction and the development of vasculitis. (A) Schematic of BMC mice generation using WT and MyD88 $8^{-1-}$ mice. (B) Vasculitis scores were assessed 28 days after CAWS injection into 8-week-recon-

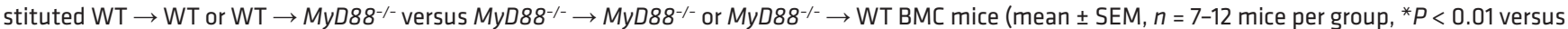
WT). (C) H\&E staining of aortic root lesions isolated from the indicated BMC mice on day 28. Scale bars: $400 \mu \mathrm{m}$. (D and E) Mouse aortic endothelial cells were stimulated with IL-1 $13(10 \mathrm{ng} / \mathrm{mL})$ for 18 hours and chemokine protein levels in the culture supernatant were measured by ELISA (mean \pm SEM, $n=3$, ${ }^{*} P<0.0001$ versus unstimulated) (D). The expression levels of adhesion molecules were assessed by flow cytometry (E). One representative of 3 independent experiments. (F and $\mathbf{G})$ qPCR analysis for chemokines (F) and chemokine receptors (G) in heart tissues isolated from WT, Dectin- $2^{-/-}$, or $I L 1 a^{-/-} I L 1 b^{-/-}$ mice 28 days after CAWS injection (mean \pm SEM, $n=4-6$ mice per group, ${ }^{*} P<0.05$ versus $W T$, ${ }^{*} P<0.01$ versus WT). $P$ values were calculated using unpaired 2-tailed Student's $t$ test (D) or 1-way ANOVA with Dunnett's post hoc test (B, F, and $\mathbf{G}$ ).

IL-1 $\beta$ production in the heart and the induction of vasculitis. Our findings indicate that iMos recruited by CCL2-producing cardiac TRMs differentiate into Mo-DCs in the middle phase of vasculitis, and produce IL-1 $\beta$ to propel neutrophilic inflammation in the late phase. This multistep immune cell mobilization system might explain the relatively long period of time (28 days) for establishing full vasculitis in this model.

GM-CSF production from radioresistant cardiac fibroblasts has also been shown to be required for CAWS-induced vasculitis (25). Because Dectin-2 expression is restricted to myeloid cells (44), it is likely that cardiac fibroblasts are not directly responding to CAWS, but instead are stimulated by cytokines, such as IL-1 $\beta$, to produce GM-CSF. As IL-1 $\beta$ is known to be a classical inducer of GM-CSF in physiological and pathological conditions (45), and IL-1 $\beta$ promotes GM-CSF production from fibroblasts (46), IL-1 $\beta$ is a potential upstream stimulator of fibroblast-derived GM-CSF production. However, Stock et al. have argued that IL-1 signaling is not essential for the development of cardiac vasculitis (25). Their conclusion was based on their findings that IL-1r1-deficient mice had no impairment in neutrophil and monocyte infiltration into the heart 1 day after CAWS injection, and that Anakinra, an IL-1R antagonist protein, did not attenuate CAWS-induced vasculitis. In contrast, we have found that $I L 1 a^{--} I L 1 b^{-/-}$mice were completely protected from vasculitis by histological analysis on day 28 . A possible explanation for this discrepancy is that Stock et al. did not analyze the hearts of $I L-1 r^{-/-}$mice histopathologically on day 28 .
It is likely that IL-1 is not involved in the initial inflammatory cell recruitment on day 1 , but instead is required for the establishment of vasculitis in the late phase. Further, Anakinra has a short halflife (4-6 hours) (47), and therefore is usually administered once or twice daily for the treatment of inflammatory diseases in humans and mice. Evidence from preclinical models of disease indicate that the therapeutic effectiveness of Anakinra is crucially dependent on the continuous saturation of IL-1Rs (48). Therefore, the 3 times a week dosing of Anakinra by Stock et al. may not have been sufficient to neutralize all of IL-1 activity in the model. Supporting a role for IL-1 in KD-like vasculitis, IL-1 $\beta$ has been shown to be required for the LCWE-induced mouse model of KD (49), and $\mathrm{IL}-1 \mathrm{ra}^{-/}$mice spontaneously develop aortitis (50).

Growing experimental evidence supports a paradigm of outside-in inflammation in vasculitis, in which vascular inflammation is initiated and perpetuated in the adventitia and progresses inward to the intima (51). In CAWS-induced vasculitis, it has been demonstrated that vascular inflammation starts from the neovascularization of the vasa vasorum in adventitia of the aorta followed by inflammatory cell accumulation (52). This is consistent with our observation that, following deposition of CAWS in the aortic root, TRMs residing in the adventitia initiate vascular inflammation. Clinically, KD is classified as a medium-vessel vasculitis, as small vessels are not commonly affected (53). In contrast, anti-neutrophil cytoplasmic antibody-associated vasculitis (AAV) is classified as a small vessel vasculitis because small vessels, and 
not medium-sized vessels, are commonly involved in this disease. Interestingly, the concept of pathogen-triggered activation of immune cells is accepted as one of the major triggers of AAV (54). Our finding that the location of antigen deposition determines the site of vascular inflammation in the CAWS model could provide a clue to solve the long-standing question of why specific vasculitis syndromes occur in specific vessels and not in all vessels and in all organs throughout the body.

Some limitations in the present study need to be considered. First, although the CAWS-induced vasculitis model is considered a representative animal model of $\mathrm{KD}(11,12,55)$, like all other animal disease models, it is not identical to the human disease, especially since the trigger for the development of $\mathrm{KD}$ is not known. However, the distribution of arterial lesions and the histological features of the vascular lesions in this model are similar to those in $\mathrm{KD}$, which are both characterized by necrotizing vasculitis with granulomatous inflammation (11). The CAWS-induced model is suitable for studying the mechanisms by which PAMPs can induce vascular inflammation, which may be of relevance to human vasculitis, but caution is needed when applying the results from mouse models directly to human disease. Second, we focused mainly on the role of myeloid cell subsets, but did not evaluate other immune cells, such as T cells, B cells, and NK cells. Taking into account the time lag between CAWS administration and established vasculitis, it is presumed that in addition to the innate immune response initiated by Dectin-2, there is also activation of the adaptive immune response that likely contributes to cardiac inflammation. Third, we have tested CD11 $\mathrm{c}^{+}$cell-specific Syk deletion in order to assess the contribution of Dectin- 2 signaling in CD11 $\mathrm{c}^{+}$cells, as Dectin-2-floxed mice are not available. However, another CLR, Mincle, also interacts with FcR $\gamma$ to activate Syk (56). As Mincle also recognizes mannose-rich Candida structures, Syk deletion could also influence Mincle-mediated signaling in vivo. Fourth, we do not understand why i.p.-injected CAWS is preferentially transported and or captured in the aortic root area of the heart and further study will be required to delineate the mechanism.

Increasing numbers of studies have implicated infection as risk factors for KD (8). Our findings demonstrate that Candida PAMPs initiate arteritis by activating TRMs in the aortic root and inducing chemokine and cytokine production in a mouse model of vasculitis resembling KD. Several case reports have shown promising results using the IL-1R antagonist Anakinra to treat KD (57, 58) and new therapeutics that target the CCL2/CCR2 chemokine axis are currently in clinical trials for cancer $(59,60)$. Finally, Syk inhibitors are available and have been in clinical trials for the treatment of rheumatoid arthritis (61). Thus, the critical pathways that we have identified that mediate CAWS-induced vasculitis can be tested as potential new therapeutic targets for combating KD.

\section{Methods}

Mice. LysM-GFP (28), Ccr2 ${ }^{R F P / R F P}$ (29), Dectin-1-/- (62), Dectin-2-/(20), IL1a $a^{-/-} I_{L 1 b^{-/-}}$(63), Card9-/- (64), and pIL1-DsRed (34) mice were maintained in our laboratory. WT C57BL/6 and congenic CD 45.1 $1^{+}$mice were purchased from Charles River Laboratories.

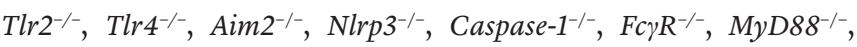
$C c l 2-R F P^{f l / f l}, S y k^{f l / f l}$, and CD11c-Cre mice were purchased from The Jackson Laboratory. CD11cCre-Sy $k^{f l f l}$ mice were generated by cross- ing $S y k^{f l / l}$ mice with $C D 11 c \mathrm{Cre}^{+} S y k^{f l / l}$ mice. All the mice were in the C57BL/6 genetic background.

Induction of CAWS-induced arteritis. CAWS was prepared from C. albicans strain IFO1385, using a previously described method (65). To induce vasculitis, CAWS (1 mg) was injected i.p. into the mice once daily for 5 days. Hearts were perfused with PBS and fixed with $10 \%$ formalin. Multiple paraffin-embedded $5-\mu \mathrm{m}$ sections were prepared and stained with hematoxylin and eosin (H\&E). To quantitatively evaluate vascular inflammation, each of 5 areas ( 3 aortic root areas and both coronary arteries) was scored 0-3 according to the classification system for the areas of cellular infiltration: (a) aortic root (score 0 for no inflammation; 1 , cell infiltration $<100 \mu \mathrm{m}$ in diameter; 2, 100-199 $\mu \mathrm{m}$ in diameter; $3, \geq 200 \mu \mathrm{m}$ in diameter); (b) coronary arteries (score 0 for no inflammation; 1 , cell infiltration $<50 \mu \mathrm{m}$ in diameter; 2, 50-99 $\mu \mathrm{m}$ in diameter; $3, \geq 100 \mu \mathrm{m}$ in diameter). The severity of arteritis in each mouse was defined as the sum of the scores of the 5 segments (maximum possible score of 15).

Parabiosis. CD 45.2 ${ }^{+}$C57BL/6 mice and naive CD $45.1^{+}$C57BL/6 mice underwent hair removal along opposite lateral flanks with the use of hair clippers and depilatory cream. Skin was then wiped clean of fur with $70 \%$ alcohol prep pads and Betadine solution. Mirrored incisions were then made on the lateral aspects of both mice. Sutures (4.0) were placed around the olecranon joints as well as the knee joints of both mice to secure the upper and lower extremities, respectively. Dorsal and ventral skin was approximated with the use of 4.0 sutures and surgical staples to conjoin the mice. At the end of the surgery, mice received subcutaneous enrofloxacin antibiotic as well as buprenorphine and flunixin for pain control. Enrofloxacin antibiotic was subsequently administered via drinking water. Subcutaneous buprenorphine and flunixin was administered as needed every 12 hours for 48 hours. Recirculation was assessed in peripheral blood 4 weeks after surgery.

In vivo adoptive cell transfer. BMDMs were negatively isolated using a mouse monocyte isolation kit (Stem Cell Technologies) from LysMGFP or Ccr2 $2^{\text {RFP/RFP }}$ mice. The cells were counted and resuspended in PBS containing $1 \%$ FCS at $0.5 \times 10^{5}$ cells $/ 100 \mu \mathrm{L}$. For competitive adoptive transfer experiments, the cells were intravenously injected at a 1:1 ratio into WT recipient mice ( $200 \mu \mathrm{L} /$ mouse) along with i.p. CAWS (1 mg) injection (Figure 2C). Twenty hours after cell transfer, peripheral blood and hearts of recipient mice were analyzed. The proportion of donor-derived $\mathrm{GFP}^{+}$and $\mathrm{RFP}^{+}$cells in the heart was detected by flow cytometry. For tracking the transferred BMDMs, LysM-GFP mouse BMDMs were intravenously injected at $0.5 \times 10^{5}$ per mouse, once daily for 5 days along with i.p. injection of CAWS (1 mg) (Figure 2F). GFP ${ }^{+}$ cells before adoptive transfer and those recovered from heart 7 days after initial CAWS injection were analyzed by flow cytometry.

Immunohistochemistry. Paraffin-embedded mouse hearts were deparaffinized and then immersed in citrate buffer (F4/80) or Borg decloaker (Ly6G/Ly6C) for 45 minutes at $95^{\circ} \mathrm{C}-97^{\circ} \mathrm{C}$, removed from heat, and kept at room temperature for 20 minutes. Endogenous peroxidase activity was blocked by incubation in endogenous peroxidase and alkaline phosphatase blocking solution (Vector Laboratories) for 10 minutes. Sections were then blocked with $5 \%$ normal goat serum for 60 minutes and stained with rabbit anti-F4/80 polyclonal antibody (Cell Signaling Technology) or rat anti-Ly6G/Ly6C mAb (Novus Bio) overnight at $4^{\circ} \mathrm{C}$. Antibody binding was amplified using SignalStain Boost IHC Detection Reagent (Cell Signaling Technology) or ImmPRESS 
polymer reagents (Vector Laboratories) and developed with DAB. The slides were examined using a bright-field microscope (Carl Zeiss).

Immunofluorescence microscopy. OCT-embedded tissue sections (7 $\mu \mathrm{m}$ thick) from frozen heart tissue were prepared. Sections were then blocked with protein block (DakoCytomation) for 15 minutes and stained with rat anti-CD11b biotinylated antibody (BioLegend), Armenian hamster anti-CD11c biotinylated antibody (BioLegend), rat anti-Ly6 G biotinylated antibody (BioLegend), or rat anti-Dectin-2 antibody (Bio-Rad) for 60 minutes at $25^{\circ} \mathrm{C}$. Streptavidin-FITC (BD Biosciences) or Alexa Fluor 488-conjugated goat anti-rat IgG antibody (Invitrogen) was used as a secondary antibody and incubated for 30 minutes at $25^{\circ} \mathrm{C}$. The slides were examined using a fluorescence microscope (Carl Zeiss).

Generation of BMC mice. BMC mice were generated according to established protocols (66). Eight weeks after reconstitution, BMC mice were used for experiments. The recovery of blood cell counts at 8 weeks after irradiation was confirmed by WBC differential count. FACS analysis of CD45.1 and CD45.2 expression on BM-derived blood cells was used to control for chimerism, exploiting the congenic expression of the CD45.1 allele in WT mice versus CD45.2 expression in $I L 1 a^{-/-} \mathrm{IL} \mathrm{b}^{-/-}$or $\mathrm{MyD} 88^{-/-}$mice. Staining reagents used were antiCD45.1-FITC and anti-CD45.2-APC (BioLegend). BMC mice were used if $95 \%$ or more of leukocytes were of donor origin.

$B M D C$ preparation. Femurs were removed from the mice and the $\mathrm{BM}$ was flushed out and plated in petri dishes with media containing GM-CSF (20 ng/mL; PeproTech). After 7 days in culture, BMDCs $(1 \times$ $10^{6}$ cells $/ \mathrm{mL}$ ) were plated in 6- or 96-well plates and then stimulated with CAWS $(100 \mu \mathrm{g} / \mathrm{mL})$, LPS $(1 \mu \mathrm{g} / \mathrm{mL}$; Sigma-Aldrich), or MUCs $(100 \mu \mathrm{g} / \mathrm{mL}$; Invivogen) for 18 hours. Cell supernatants were analyzed for IL-1 $\beta$ by ELISA (R\&D Systems). Cell lysates and concentrated culture supernatants were used for immunoblot analysis.

Flow cytometry. The hearts were minced and digested with $450 \mathrm{U} /$ $\mathrm{mL}$ collagenase I, $125 \mathrm{U} / \mathrm{mL}$ collagenase XI, $60 \mathrm{U} / \mathrm{mL}$ DNase I, and $60 \mathrm{U} / \mathrm{mL}$ hyaluronidase (Sigma-Aldrich) in PBS for 1 hour at $37^{\circ} \mathrm{C}$ with shaking (67). A cell suspension was obtained by mashing the tissue through a $70-\mu \mathrm{m}$ strainer. Single cells were incubated with anti-mouse CD16/32 (93, TruStain fcX, BioLegend) to block Fc receptors and then stained with Fixable Viability Dye eF780 (eBioscience) to identify dead cells and fluorochrome-conjugated anti-mouse Abs as indicated in Supplemental Table 1. In preliminary experiments, CD $45 \mathrm{mAb}$ was administered intravenously 3 minutes before harvesting to distinguish intravascular leukocytes that remain even after vigorous flushing from truly intraparenchymal leukocytes. As has been described (68), in contrast to other organs like the lung, we only found that approximately $10 \%$ of the leukocytes stained with intravenous CD $45 \mathrm{mAb}$ (data not shown). Therefore, we did not routinely perform this technique for this study that exclusively focused on the heart.

$q P C R$. Tissue was isolated and homogenized in TRIzol (SigmaAldrich) and RNA was isolated according to the manufacturer's instructions. Purified RNA was then converted to cDNA by reverse transcription (TaqMan Reverse Transcriptase Reagents, Themo Fisher Scientific). qPCR in the presence of SYBR Green (FastStart Essential DNA Green Master Mix, Roche) was performed on a LightCycler 96 Instrument (Roche) and normalized to $\beta_{2}$ microglobulin $(\beta 2 m)$. The sequences for the qPCR primers can be found in Supplemental Table 2.

Quantitative immunofluorescence analysis of NF- $\kappa$ B nuclear translocation. BMDCs were plated and cultured on glass coverslips coated with recombinant ICAM-1 $(2.5 \mu \mathrm{g} / \mathrm{mL}$; BioLegend $)$ at a density of $2 \times$ $10^{5}$ cells per well in 8-well plates. The cells were treated with CAWS $(100 \mu \mathrm{g} / \mathrm{mL})$, LPS $(1 \mu \mathrm{g} / \mathrm{mL}$; Sigma-Aldrich), or PBS for 60 minutes at $37^{\circ} \mathrm{C}$. Then the cells were fixed with $4 \%$ formalin in PBS for $15 \mathrm{~min}$ utes at room temperature. They were then permeabilized with PBS containing $1 \%$ Triton $\mathrm{X}-100$ for 10 minutes at room temperature. To block the nonspecific binding of the antibodies, samples were incubated with protein block (DakoCytomation) for 15 minutes at room temperature. Rabbit mAb for NF-кB p65 (1:400; Cell Signaling Technology) was added to the samples and incubated 18 hours at $4^{\circ} \mathrm{C}$. Then the cells were incubated with anti-rabbit secondary antibody conjugated with Alexa Fluor 546 (Invitrogen) for 30 minutes at room temperature. Nuclear/cytoplasmic ratios of NF-кB p65 were analyzed using a fluorescence microscope (Carl Zeiss).

In vitro cardiac macrophage stimulation. Single-cell suspensions derived from heart were passed through a $30-\mu \mathrm{m}$ cell strainer twice followed by incubation with anti-F4/80 microbeads (Miltenyi Biotec). Beads with bound $\mathrm{F} 4 / 80^{+}$cells were magnetically isolated by magneticactivated cell sorting (MACS). $\mathrm{F} 4 / 80^{+}$cells were plated at a density of $1 \times 10^{5}$ cells per well in 96-well plates. The cells were treated with CAWS $(10 \mu \mathrm{g} / \mathrm{mL})$ or PBS for 18 hours at $37^{\circ} \mathrm{C}$. Cell-free culture supernatants were assayed for CCL2 (R\&D Systems) by ELISA.

In vitro MAEC stimulation. C57BL/6 primary MAECs were purchased from Cell Biologics. The cells were seeded at $1 \times 10^{5}$ in 24 -well plates and cultured in endothelial cell medium (Cell Biologics) at $37^{\circ} \mathrm{C}$. Cultures were stimulated with recombinant IL-1 $\beta$ (20 ng/mL; BioLegend) for 18 hours. Then the cells were stained for flow cytometry and the chemokine levels in the culture supernatants were assessed by Multi-Analyte ELISArray Kit (Qiagen).

In vitro mouse cardiac fibroblast stimulation. C57BL/6 mouse primary cardiac fibroblasts were purchased from Cell Biologics. The cells were seeded at $2 \times 10^{4}$ per well in 96-well plates and cultured in complete DMEM at $37^{\circ} \mathrm{C}$. Cultures were stimulated with CAWS $(10 \mu \mathrm{g} / \mathrm{mL}$, $100 \mu \mathrm{g} / \mathrm{mL}$, or $1 \mathrm{mg} / \mathrm{mL}$ ), recombinant IL-1 $\beta$ (50 ng/mL; BioLegend), recombinant TNF- $\alpha$ (50 ng/mL; PeproTech), or LPS ( $50 \mathrm{mg} / \mathrm{mL}$; SigmaAldrich) for 18 hours. Cell lysates were used for qPCR analysis. Cell-free culture supernatants were assayed for CCL2 (R\&D Systems) by ELISA.

Statistical analysis. Data were analyzed using Prism 7 (GraphPad Software Inc.), and results were expressed as the mean \pm SEM. $P$ values for multiple groups were calculated using ordinary 1-way ANOVA with Dunnett's post hoc test or Tukey's multiple-comparisons test as appropriate. Means between 2 groups were compared with unpaired 2-tailed Student's $t$ test. A P value less than 0.05 was considered significant.

Study approval. All experiments described herein were approved by the IACUC of Massachusetts General Hospital.

\section{Author contributions}

CM designed and performed most experiments, analyzed and interpreted data, and contributed to writing the manuscript. YM performed and analyzed BMC and in vivo adoptive transfer experiments. LBM assisted in the qPCR, ELISA, and IHC experiments. JL provided technical assistance for the flow cytometry and qPCR experiments. RAR generated parabiotic mice. NNM and NO provided CAWS. YI provided IL1 $a^{-/-} I L 1 b^{-/-}$mice, Dectin $-^{-/-}$mice, and Dectin- $2^{-/-}$mice. TK supervised the project. ADL provided overall project supervision, contributed to the design of the experiments, and wrote the manuscript. 


\section{Acknowledgments}

This work is funded by NIH grant R01AI050892 and Rheumatology Research Foundation (to ADL), and Japan Heart Association and Japan Blood Products organization (to $\mathrm{CM}$ ). The authors thank N. Barrett (Brigham and Women's Hospital, Boston, Massachusetts, USA) for Dectin-1- and Dectin-2-deficient mice, R. Xavier (Massachusetts General Hospital) for Card9-deficient mice, and S. Lacroix (Laval University, Québec City, Canada) for pIL1-
DsRed reporter mice. We would also like to thank A. Sato and S. Ji for their technical support.

Address correspondence to: Andrew D. Luster, Center for Immunology and Inflammatory Diseases, Division of Rheumatology, Allergy and Immunology, Massachusetts General Hospital, Building 149-8.304, 13th Street, Charlestown, Massachusetts 02129, USA. Phone: 617.726.5710; Email: aluster@mgh.harvard.edu.
1. Kawasaki T. [Acute febrile mucocutaneous syndrome with lymphoid involvement with specific desquamation of the fingers and toes in children]. Arerugi. 1967;16(3):178-222.

2. Taubert KA, Rowley AH, Shulman ST. Seven-year national survey of Kawasaki disease and acute rheumatic fever. Pediatr Infect Dis J. 1994;13(8):704-708.

3. Takahashi K, Oharaseki T, Yokouchi Y, Hiruta N, Naoe S. Kawasaki disease as a systemic vasculitis in childhood. Ann Vasc Dis. 2010;3(3):173-181.

4. Kato H, et al. Long-term consequences of Kawasaki disease. A 10- to 21-year follow-up study of 594 patients. Circulation. 1996;94(6):1379-1385.

5. Newburger JW, et al. A single intravenous infusion of gamma globulin as compared with four infusions in the treatment of acute Kawasaki syndrome. N Engl JMed. 1991;324(23):1633-1639.

6. Greco A, et al. Kawasaki disease: an evolving paradigm. Autoimmun Rev. 2015;14(8):703-709.

7. Burns JC, Glodé MP. Kawasaki syndrome. Lancet. 2004;364(9433):533-544.

8. Wang CL, Wu YT, Liu CA, Kuo HC, Yang KD. Kawasaki disease: infection, immunity and genetics. Pediatr Infect Dis J. 2005;24(11):998-1004.

9. Xie X, Shi X, Liu M. The roles of genetic factors in Kawasaki disease: A systematic review and meta-analysis of genetic association studies. Pediatr Cardiol. 2018;39(2):207-225.

10. Luzina IG, Handwerger BS. Lessons from animal models of vasculitis. Curr Rheumatol Rep. 2000;2(5):369-375.

11. Takahashi K, Oharaseki T, Wakayama M, Yokouchi Y, Naoe S, Murata H. Histopathological features of murine systemic vasculitis caused by Candida albicans extract--an animal model of Kawasaki disease. Inflamm Res. 2004;53(2):72-77.

12. Miyabe C, et al. Am80, a retinoic acid receptor agonist, ameliorates murine vasculitis through the suppression of neutrophil migration and activation. Arthritis Rheum. 2013;65(2):503-512.

13. Lehman TJ, Walker SM, Mahnovski V, McCurdy D. Coronary arteritis in mice following the systemic injection of group B Lactobacillus casei cell walls in aqueous suspension. Arthritis Rheum. 1985;28(6):652-659.

14. Nishio H, et al. Nod1 ligands induce site-specific vascular inflammation. Arterioscler Thromb Vasc Biol. 2011;31(5):1093-1099.

15. Rodó X, et al. Association of Kawasaki disease with tropospheric wind patterns. Sci Rep. 2011;1:152.

16. Rodó X, et al. Tropospheric winds from northeastern China carry the etiologic agent of Kawasaki disease from its source to Japan. Proc Natl Acad Sci U S A. 2014;111(22):7952-7957.
17. El-Askary H, LaHaye N, Linstead E, Sprigg WA, Yacoub M. Remote sensing observation of annual dust cycles and possible causality of Kawasaki disease outbreaks in Japan. Glob Cardiol Sci Pract. 2017;2017(3):e201722.

18. Ishibashi K, Fukazawa R, Miura NN, Adachi Y, Ogawa S, Ohno N. Diagnostic potential of antibody titres against Candida cell wall $\beta$-glucan in Kawasaki disease. Clin Exp Immunol. 2014;177(1):161-167.

19. Takeuchi O, Akira S. Pattern recognition receptors and inflammation. Cell. 2010;140(6):805-820.

20. Saijo S, et al. Dectin-2 recognition of alpha-mannans and induction of Th17 cell differentiation is essential for host defense against Candida albicans. Immunity. 2010;32(5):681-691.

21. Brown GD. Dectin-1: a signalling non-TLR pattern-recognition receptor. Nat Rev Immunol. 2006;6(1):33-43.

22. McGreal EP, et al. The carbohydrate-recognition domain of Dectin-2 is a C-type lectin with specificity for high mannose. Glycobiology. 2006;16(5):422-430.

23. Drummond RA, Saijo S, Iwakura Y, Brown GD. The role of Syk/CARD9 coupled C-type lectins in antifungal immunity. Eur J Immunol. 2011;41(2):276-281.

24. Netea MG, et al. Immune sensing of Candida albicans requires cooperative recognition of mannans and glucans by lectin and Toll-like receptors. JClin Invest. 2006;116(6):1642-1650.

25. Stock AT, Hansen JA, Sleeman MA, McKenzie BS Wicks IP. GM-CSF primes cardiac inflammation in a mouse model of Kawasaki disease. J Exp Med. 2016;213(10):1983-1998.

26. Tamoutounour S, et al. Origins and functional specialization of macrophages and of conventional and monocyte-derived dendritic cells in mouse skin. Immunity. 2013;39(5):925-938.

27. Domínguez-Andrés J, Feo-Lucas L, Minguito de la Escalera M, González L, López-Bravo M, Ardavín C. Inflammatory Ly6C. Immunity. 2017;46(6):1059-1072.e4.

28. Faust N, Varas F, Kelly LM, Heck S, Graf T. Insertion of enhanced green fluorescent protein into the lysozyme gene creates mice with green fluorescent granulocytes and macrophages. Blood. 2000;96(2):719-726.

29. Saederup N, et al. Selective chemokine receptor usage by central nervous system myeloid cells in CCR2-red fluorescent protein knock-in mice. PLOS ONE. 2010;5(10):e13693.

30. Shi C, et al. Bone marrow mesenchymal stem and progenitor cells induce monocyte emigration in response to circulating Toll-like receptor ligands. Immunity. 2011;34(4):590-601.
31. Netea MG, Van Der Graaf CA, Vonk AG, Verschueren I, Van Der Meer JW, Kullberg BJ. The role of Toll-like receptor (TLR) 2 and TLR4 in the host defense against disseminated candidiasis. J Infect Dis. 2002;185(10):1483-1489.

32. Saijo K, et al. Essential role of Src-family protein tyrosine kinases in NF-kappaB activation during B cell development. Nat Immunol. 2003;4(3):274-279.

33. Caton ML, Smith-Raska MR, Reizis B. NotchRBP-J signaling controls the homeostasis of CD8 dendritic cells in the spleen. J Exp Med. 2007;204(7):1653-1664.

34. Matsushima H, Ogawa Y, Miyazaki T, Tanaka H, Nishibu A, Takashima A. Intravital imaging of IL-1beta production in skin. J Invest Dermatol. 2010;130(6):1571-1580.

35. Strowig T, Henao-Mejia J, Elinav E, Flavell R. Inflammasomes in health and disease. Nature. 2012;481(7381):278-286.

36. Jo EK, Kim JK, Shin DM, Sasakawa C. Molecular mechanisms regulating NLRP3 inflammasome activation. Cell Mol Immunol. 2016;13(2):148-159.

37. Shinohara $\mathrm{H}$, et al. Beta-mannosyl linkages negatively regulate anaphylaxis and vasculitis in mice, induced by CAWS, fungal PAMPS composed of mannoprotein-beta-glucan complex secreted by Candida albicans. Biol Pharm Bull. 2006;29(9):1854-1861.

38. Davies LC, Jenkins SJ, Allen JE, Taylor PR. Tissue-resident macrophages. Nat Immunol. 2013;14(10):986-995.

39. Gosselin D, et al. Environment drives selection and function of enhancers controlling tissue-specific macrophage identities. Cell. 2014;159(6):1327-1340.

40. Lavin Y, et al. Tissue-resident macrophage enhancer landscapes are shaped by the local microenvironment. Cell. 2014;159(6):1312-1326.

41. Epelman S, et al. Embryonic and adult-derived resident cardiac macrophages are maintained through distinct mechanisms at steady state and during inflammation. Immunity. 2014;40(1):91-104.

42. Taylor PR, Martinez-Pomares L, Stacey M, Lin $\mathrm{HH}$, Brown GD, Gordon S. Macrophage receptors and immune recognition. Annu Rev Immunol. 2005;23:901-944.

43. Martinez HG, et al. Important role of CCR2 in a murine model of coronary vasculitis. BMC Immunol. 2012;13:56

44. Taylor PR, Reid DM, Heinsbroek SE, Brown GD, Gordon S, Wong SY. Dectin-2 is predominantly myeloid restricted and exhibits unique activation-dependent expression on maturing inflammatory monocytes elicited in vivo. Eur J 
Immunol. 2005;35(7):2163-2174.

45. Seelentag WK, Mermod JJ, Montesano R, Vassalli P. Additive effects of interleukin 1 and tumour necrosis factor-alpha on the accumulation of the three granulocyte and macrophage colony-stimulating factor mRNAs in human endothelial cells. ЕМВO J. 1987;6(8):2261-2265.

46. Darrieutort-Laffite C, et al. IL-1 $\beta$ and TNF $\alpha$ promote monocyte viability through the induction of GM-CSF expression by rheumatoid arthritis synovial fibroblasts. Mediators Inflamm. 2014;2014:241840.

47. Braddock M, Quinn A. Targeting IL-1 in inflammatory disease: new opportunities for therapeutic intervention. Nat Rev Drug Discov. 2004;3(4):330-339.

48. Bendele A, McAbee T, Sennello G, Frazier J, Chlipala E, McCabe D. Efficacy of sustained blood levels of interleukin-1 receptor antagonist in animal models of arthritis: comparison of efficacy in animal models with human clinical data. Arthritis Rheum. 1999;42(3):498-506.

49. Wakita D, et al. Role of interleukin-1 signaling in a mouse model of Kawasaki disease-associated abdominal aortic aneurysm. Arterioscler Thromb Vasc Biol. 2016;36(5):886-897.

50. Matsuki T, et al. Involvement of tumor necrosis factor-alpha in the development of $\mathrm{T}$ cell-dependent aortitis in interleukin-1 receptor antagonist-deficient mice. Circulation. 2005;112(9):1323-1331.

51. Stenmark KR, et al. The adventitia: essential regulator of vascular wall structure and function. Annu Rev Physiol. 2013;75:23-47.
52. Hamaoka-Okamoto A, et al. The involvement of the vasa vasorum in the development of vasculitis in animal model of Kawasaki disease. Pediatr Rheumatol Online J. 2014;12:12.

53. Jennette JC, et al. 2012 revised International Chapel Hill Consensus Conference Nomenclature of Vasculitides. Arthritis Rheum. 2013;65(1):1-11.

54. Nakazawa D, Masuda S, Tomaru U, Ishizu A. Pathogenesis and therapeutic interventions for ANCA-associated vasculitis. Nat Rev Rheumatol. 2019;15(2):91-101.

55. Miyabe C, et al. A sphingosine 1-phosphate receptor agonist ameliorates animal model of vasculitis. Inflamm Res. 2017;66(4):335-340.

56. Yamasaki S, Ishikawa E, Sakuma M, Hara H, Ogata K, Saito T. Mincle is an ITAM-coupled activating receptor that senses damaged cells. Nat Immunol. 2008;9(10):1179-1188.

57. Guillaume MP, Reumaux H, Dubos F. Usefulness and safety of anakinra in refractory Kawasaki dis ease complicated by coronary artery aneurysm. Cardiol Young. 2018;28(5):739-742.

58. Kone-Paut I, et al. The use of interleukin 1 receptor antagonist (anakinra) in Kawasaki disease: A retrospective cases series. Autoimmun Rev. 2018;17(8):768-774.

59. Noel M, et al. 750P Phase Ib study of PF-04136309 (an oral CCR2 inhibitor) in combination with nab-paclitaxel/gemcitabine in firstline treatment of metastatic pancreatic adenocarcinoma. Ann Oncol. 2017; 28(suppl_5):mdx369.132-mdx369.132.

60. Noel MS, et al. Orally administered CCR2 selective inhibitor CCX872-b clinical trial in pancreat- ic cancer. JClin Oncol. 2017;35(4_suppl):276.

61. Kunwar S, Devkota AR, Ghimire DK. Fostamatinib, an oral spleen tyrosine kinase inhibitor, in the treatment of rheumatoid arthritis: a meta-analysis of randomized controlled trials. Rheumatol Int. 2016;36(8):1077-1087.

62. Saijo S, et al. Dectin-1 is required for host defense against Pneumocystis carinii but not against Candida albicans. Nat Immunol. 2007;8(1):39-46.

63. Horai R, et al. Production of mice deficient in genes for interleukin (IL)-1alpha, IL-1beta, IL-1alpha/beta, and IL-1 receptor antagonist shows that IL-1beta is crucial in turpentineinduced fever development and glucocorticoid secretion. JExp Med. 1998;187(9):1463-1475.

64. Hara $\mathrm{H}$, et al. The adaptor protein CARD9 is essential for the activation of myeloid cells through ITAM-associated and Toll-like receptors. Nat Immunol. 2007;8(6):619-629.

65. Uchiyama M, et al. Chemical and immunochemical characterization of limulus factor G-activating substance of Candida spp. FEMS Immunol Med Microbiol. 1999;24(4):411-420.

66. Sadik CD, Kim ND, Iwakura Y, Luster AD. Neutrophils orchestrate their own recruitment in murine arthritis through C5aR and Fc $\gamma$ R signaling. Proc Natl Acad Sci U S A. 2012;109(46):E3177-E3185.

67. Anzai A, et al. The infarcted myocardium solicits GM-CSF for the detrimental oversupply of inflammatory leukocytes. J Exp Med. 2017;214(11):3293-3310.

68. Ramos GC, et al. Myocardial aging as a T-cell-mediated phenomenon. Proc Natl Acad Sci U S A. 2017;114(12):E2420-E2429. 